\title{
A Stratification of the Null Cone Via the Moment Map
}

\section{Citation}

Mumford, David B. 1984. App. to "A stratification of the null cone via the moment map," by Linda Ness. American Journal of Mathematics 106(6): 1281-1329.

\section{Published Version}

doi: $10.2307 / 2374395$

\section{Permanent link}

http://nrs.harvard.edu/urn-3:HUL.InstRepos:3615198

\section{Terms of Use}

This article was downloaded from Harvard University's DASH repository, and is made available under the terms and conditions applicable to Other Posted Material, as set forth at http:// nrs.harvard.edu/urn-3:HUL.InstRepos:dash.current.terms-of-use\#LAA

\section{Share Your Story}

The Harvard community has made this article openly available.

Please share how this access benefits you. Submit a story.

Accessibility 


\title{
A STRATIFICATION OF THE NULL CONE VIA THE MOMENT MAP
}

\author{
By Linda NesS \\ With an Appendix by David MuMford
}

0. Introduction. Given $G \times \mathbf{V} \rightarrow \mathbf{V}$, a linear representation of a reductive group $G$, defined over $\mathbf{C}$, on a finite dimensional complex vector space $\mathbf{V}$, one may fix a maximal compact subgroup $K \subset G$ and a $K$ invariant hermitian inner product on $\mathbf{V}$.

From this data, one can define a moment map, as in symplectic geometry,

$$
m: \mathbf{P}(\mathbf{V}) \rightarrow \operatorname{Lie} G^{*} / \operatorname{Lie} K^{*} \approx i i^{*} \quad \text { by } m(x)=\frac{1}{\|v\|^{2}} d_{e}\|g \cdot v\|^{2}
$$

and the square-norm of the moment map

$$
\|m\|^{2}: \mathbf{P}(\mathbf{V}) \rightarrow \mathbf{R} \quad\|m\|^{2}(x)=\|m(x)\|^{2} .
$$

Here $v \in \mathbf{V}-\{0\}$ lies over $x$, and the differential of $G \rightarrow \mathbf{R} g \rightarrow\|g \cdot v\|^{2}$ is computed at the identity $e \in G$. Also $\|m(x)\|^{2}$ is computed with respect to an ad- $K$ invariant inner product on $i{ }^{*}$. The moment map is $K$-equivariant and its component functions are Hamiltonians for the canonical vector fields on $\mathbf{P}(\mathbf{V})$. The norm-square $\|m\|^{2}$ is a $K$-invariant function on $\mathbf{P}(\mathbf{V})$.

The first observation $[\mathrm{K}-\mathrm{N}]$ is the orbit $G \cdot v$ is closed if and only if $m(g \cdot x)=0$ for some $g \in G$ if and only if $\|m\|^{2}(g \cdot x)=0$ for some $g \in G$. Here $v \neq 0$ lies over $x$.

In this paper, I apply the moment map to the main problem of Geometric Invariant Theory-understanding the $G$-orbit space of the action of a reductive group on a complex projective variety. I have restricted attention to the core case-where the variety is $\mathbf{P}(\mathbf{V})$ - so that I did not have to introduce the notion of linearization of an invertible sheaf-a common initial source of confusion. The results are easily generalized. 
If one wants to construct a quotient of $\mathbf{P}(\mathbf{V})$ by $G$, one must first throw out the variety of $N \subset \mathbf{P}(\mathbf{V})$, where all of the $G$-invariant homogeneous polynomials vanish. This variety is called the null-cone $N$; it consists of the "unstable points." Mumford proved in [M] that the quotient of the complement $\mathbf{P}(\mathbf{V})-N=\mathbf{P}(\mathbf{V})_{s s}$ (the semistable points) by extended $G$ equivalence is a projective variety $\mathbf{P}(\mathbf{V})_{s s / G}$. In Section 2 it is shown that this is the "Marsden-Weinstein reduction" of symplectic geometry.

In Sections 3 through 7 the real valued function $\|m\|^{2}: \mathbf{P}(\mathbf{V}) \rightarrow \mathbf{R}$ is studied. The trajectories of the gradient flow of $\|m\|^{2}$ are easily seen to be tangent to the $G$-orbits. The limit set of the gradient flow of $\|m\|^{2}$ is, of course, the set of critical points of $\|m\|^{2}$. The main result on the critical locus of $\|m\|^{2}$ is

TheOREM 6.2. Let $\left.\|m\|^{2}\right|_{G \cdot x}$ denote the restriction of $\|m\|^{2}$ to the G-orbit of $x$ in $\mathbf{P}(\mathbf{V})$. If $d\|m\|^{2}(x)=0$, then

(i) $\left.\|m\|^{2}\right|_{G \cdot x}$ attains its minimum value at $x$

(ii) $\left.\|m\|^{2}\right|_{G \cdot x}$ attains its minimum value on a unique $K$-orbit.

The nonminimal critical points are all in the null one. Hence, the gradient flow of $\|m\|^{2}$ determines a stratification of the null cone $N$. A stratum $N_{\langle\alpha\rangle}$ of $N$ is the set of all points $x$ which flow into $C_{\langle\alpha\rangle}$, where $C_{\langle\alpha\rangle}$ is the set of critical points $y$ of $\|m\|^{2}$ such that $m^{*}(y) \in K \cdot \alpha$. Clearly the limit set $C_{\langle\alpha\rangle} \subset N_{\langle\alpha\rangle}$ is $K$-invariant.

Hesselink in $[\mathrm{H}]$ defined an algebraic stratification of the null cone using Kempf's adapted one-parameter subgroups. There is a natural flow via adapted one-parameter subgroups and hence, a limit set in each strata $L_{M, \alpha}$, which is invariant under the action of a "smaller" reductive group, determined by the strata.

In Section 9 we show there is a natural one-to-one correspondence between the two stratifications. The main result of Section 9 is a comparison of the two limit sets. The main result is: the quotients $C_{\langle\alpha\rangle / K}$ and $L_{M,\langle\alpha\rangle / L^{\prime}}$ are naturally isomorphic projective algebraic varieties.

The point of this result is the following: one would like to understand the space $\mathbf{P}(\mathbf{V})_{s s / G}=\mathbf{P}(\mathbf{V})-N / G$. One could do this, mimicing Bott and Atiyah in [A-B] by understanding the varieties of $C_{\langle\alpha\rangle / K} \approx L_{M,\langle\alpha\rangle / L^{\prime}}$ arising from the stratification of the null cone.

Examples are computed in Sections 8 and 10.

There has recently been a flurry of applications of moment maps to geometry. It seems that the impetus came from a thesis of Gerritt Heck- 
man $[\mathrm{He}]$ in which he proved Kostant's convexity theorem using these techniques. Guillemin and Sternberg observed that one could define a moment map in the case that we are considering and proved a more general convexity theorem [G-S]. This theorem is stated in Section 5 and a proof due to Mumford is in the appendix. Atiyah [At] has independently given a proof of the convexity theorem for the case $G=T$, a torus using Morse Theoretic techniques. Bott and Attiyah point out that the Yang Mills functional on connections on vector bundles over a Riemann surface can be interpreted as the square norm of a moment map [A-B].

Recently, I was informed that Frances Kirwin in her Oxford thesis is studying the stratification determined by the gradient flow of $\|\boldsymbol{m}\|^{2}$ in the case of reductive group acting on a smooth projective variety. For the case that the stabilizer of every semistable point is finite she announces a formula for the rational cohomology of the quotient variety $\mathbf{P}(\mathbf{V})_{s s} / G$. Her approach uses $G$-quivariant Morse-theory.

Finally, I would like to thank David Mumford for suggesting this problem to me, for several discussions of the problem, and for writing the appendix. The basic outline of Section 2 follows a lecture he gave at the Institute of Advanced Study in Princeton in March 1982.

1. The moment map on projective space. Let $G$ be a reductive group defined over $\mathbf{C}$. Let $\mathbf{V}$ be a complex vector space of finite dimension. Assume $G$ acts linearly on $\mathbf{V}$ by

$$
G \times \mathbf{V} \rightarrow \mathbf{V} \quad(g, v) \rightarrow g \cdot v .
$$

Fix a maximal compact subgroup $K \subset G$. We may assume $\mathbf{V}$ is endowed with an hermitian inner product $\langle$,$\rangle which is invariant under the action of$ $K$, so $\langle k \cdot v, k \cdot w\rangle=\langle v, w\rangle$ for all $k \in K$, and for all $v$ and $w$ in $\nabla$. As usual let $\|v\|^{2}=\langle v, v\rangle$.

We want to study how the squared length changes as $G$ moves a vector $v$ infinitesimally along its orbit. Because $G$ acts linearly, we only need study this for each $x \in \mathbf{P}(\mathbf{V})$, the projective space of lines in $\mathbf{V}$.

For each $v \in \mathbf{V}$, define

$$
\rho_{v}: G \rightarrow \mathbf{R} \quad \text { by } g \rightarrow\|g \cdot v\|^{2},
$$

Now let $d \rho_{\nu}(e)$ denote the differential of $\rho_{\nu}$ computed at the identity $\boldsymbol{e}$ of $G$. Since the inner product is $K$-invariant we may view $d \rho_{v}(e) \in \mathrm{g}^{*} / \mathfrak{f}^{*}$, where 
$\mathrm{g}^{*}$ and $\mathfrak{f}^{*}$ are the vector spaces of real-valued linear functionals on the lie algebras $\mathfrak{g}=\operatorname{Lie}(G)$ and $\mathfrak{f}=\operatorname{Lie}(K)$. Since $G$ is reductive $\mathfrak{g} \approx \mathfrak{t} \oplus i \mathfrak{i}$. Thus we may define a function

$$
m: \mathbf{P}(\mathbf{V}) \rightarrow i i^{*} \quad \text { by } x \rightarrow \frac{d \rho_{v}(e)}{\|v\|^{2}}, v \in \mathbf{V} \quad \text { over } x, v \neq 0
$$

Thus $\Pi: \mathbf{V}-\{0\} \rightarrow \mathbf{P}(\mathbf{V})$ is the usual projection map, $\Pi(v)=x$.

We will verify in this section that $m$ is a moment map (of symplectic geometry).** The key observation, which we will use throughout the paper, will be: the component functions of $m$ are easy to compute and easy to differentiate. For each $\alpha \in i f$, the component function $m_{\alpha}(x)=m(x)(\alpha)$. Thus $m_{\alpha}$ just gives the infinitesimal variation of the squared length as $v$ is moved by the real one-parameter subgroup $\exp t \alpha$, so

$$
m_{\alpha}(x)=\left.\frac{1}{\|v\|^{2}} \frac{d}{d t}\right|_{t=0}\|\exp t \alpha \cdot v\|^{2}, v \text { over } x .
$$

A moment map [G-S] is defined as follows. Suppose $M$ is a real manifold, of even dimension, endowed with a closed nondegenerate 2 form $\omega$. Suppose $K$ is a real compact Lie group which acts on the symplectic manifold $M$ preserving its symplectic structure. Thus there is a homomorphism

$$
\phi: K \rightarrow \operatorname{Aut}(M) \quad k \rightarrow \phi \quad \text { and } \quad \phi_{k}{ }^{*} \omega=\omega .
$$

For each $\alpha \in \mathfrak{f}=\operatorname{Lie}(K)$, the action of $K$ determines a canonical vector field $X_{\alpha}$ on $M ; X_{\alpha}$ is just $d \phi(\alpha)$. Note that $K$ acts on $\mathfrak{f}^{*}$, the real dual of $\mathfrak{f}$ by the coadjant action.

A map

$$
\Phi: M \rightarrow \mathfrak{f}^{*} \quad \text { is a moment map }
$$

if

(1) $\Phi$ is $K$ equivariant

(2) For each $\alpha \in \mathfrak{f}, d \Phi_{\alpha}=\omega\left(, X_{\alpha}\right)$ so $X_{\alpha}$ is the Hamiltonian vectorfield for $\Phi_{\alpha}$.

\footnotetext{
**Guillemin and Sternberg proved this in [G-S]; the verification here is, I believe, simpler. One example of a moment map is angular momentum [A].
} 
Here $\Phi_{\alpha}: M \rightarrow \mathbf{R}$ is $\Phi$ followed by evaluation at $\alpha, \Phi_{\alpha}(x)=\Phi(x)(\alpha)$, $x \in M$.

There is a natural symplectic structure on $\mathbf{P}(\mathbf{V})$. Let $\omega$ denote the real 1-1 form of a Fubini-study metric on $\mathbf{P}(\mathbf{V})$ determined by the inner product $\langle$,$\rangle on \mathbf{V}$. We will normalize so $\omega$ is computed as follows. Let $Z: W \rightarrow \mathbf{V}-$ $\{0\}$ denote a local holomorphic lifting of an open set $W \subset \mathbf{P}(\mathbf{V})$. Then on $W$

$$
\omega=2 i \partial \bar{\partial} \log \|Z\|^{2}
$$

Since $\omega$ is real nondegenerate and closed, $\omega$ is a symplectic form on $\mathbf{P}(\mathbf{V})$. Furthermore, for any automorphism $U$ of $\mathbf{P}(\mathbf{V})$ induced by a unitary transformation of $\mathbf{V}, U^{*} \omega=\omega$. Thus the action of the maximal compact subgroup $K \subset G$ preserves the symplectic structure. Note that $\omega$ is " 4 times the usual normalization for the Fubini-study metric."

For each $\alpha \in \mathfrak{g}$, there is a canonical vector field $X_{\alpha}$ on $\mathbf{P}(\mathbf{V})$ and a canonical vector field $\hat{X}_{\alpha}$ on $\mathbf{V}$. They are related by $\Pi_{*} \hat{X}_{\alpha}=X_{\alpha}$. The canonical vector fields on $\mathbf{V}$ are easily understood. Denote the action of $G$ on V by

$$
\phi: G \rightarrow G L(\mathbf{V}) .
$$

Then at $e$ in $G$

$$
d \phi_{e}: \mathfrak{g} \rightarrow \mathfrak{g} \ell(\mathbf{V})=\text { Lie } G L(\mathbf{V})
$$

For $\alpha \in \mathrm{g}$, let $T_{\alpha}=d \phi_{e}(\alpha)$. Then the canonical vector field on $\mathbf{V}$ is defined by

$$
\hat{X}_{\alpha}(v)=T_{\alpha}(v)=d \phi_{e}(\alpha)(v) .
$$

Recall that $\mathfrak{g} \ell(\mathbf{V})=i \mathfrak{H C} \oplus \mathfrak{H C}$ where $\mathfrak{H C}$ is the real vector space of Hermitian transformations of $\mathbf{V}$, relative to $\langle$,$\rangle . Thus i \mathcal{H C}$ consists of the skew Hermitian transformations of $\mathbf{V}$, so $i \mathcal{F C}$ is the Lie algebra of the unitary transformations of $\mathbf{V}$ relative to $\langle\rangle,, i \mathcal{F C}=\mathbf{V}$. Since the inner product is $K$ invariant, $d \phi_{e}(\mathfrak{f}) \subset i \mathcal{F}$. Since $d \phi_{e}$ is complex linear $d \phi_{e}(i \mathfrak{f}) \subset i \mathcal{H}$. In terms of the notation we just introduced, this is expressed as the

Facts: (i) For $\alpha \in \mathfrak{g}, T_{i \alpha}=i T_{\alpha}$

(ii) If $\alpha \in \mathfrak{t}, T_{\alpha} \in i \mathfrak{H C}$

(iii) If $\alpha \in i \mathfrak{i}, T_{\alpha} \in \mathcal{H C}$. 
The last fact (iii) implies that for each $\alpha \in i f$, there is an orthogonal decomposition of $\mathbf{V}$, namely the decomposition of $\mathbf{V}$ into eigenspaces for $T_{\alpha}$

$$
\mathbf{V}=\oplus \mathbf{V}_{i} \quad \text { for } v \quad V \text {, write } v=\Sigma v_{i}, v_{i} \in \mathbf{V}_{i}
$$

Since $T_{\alpha} V=\left.(d / d t)\right|_{t=0} \exp t \alpha \cdot v,(6)$ implies that

$$
\exp t \alpha \cdot v=\sum_{i} e^{a_{i} t} v_{i}
$$

Thus we will call the decomposition (5) the $\alpha$-weight decomposition of $V$; we will call the $a_{i}$ 's the $\alpha$-weights.

Lemma 1.1. Suppose $\alpha \in i f$. The canonical vector field $X_{\alpha}$ on $\mathbf{P}(\mathbf{V})$ vanishes at $x$ if and only if the line in $\mathrm{V}$ over $x$ is contained in one of the $\alpha$ weight spaces if and only if $\exp t \alpha \cdot x=x$ or $x \in G_{x}$.

Proof. Identify $\mathbf{V}$ with the tangent space to $\mathbf{Y}$ at $v$. Then the kernel of $\Pi_{*}$ at $v$ is the line through origin containing $v$. For $v$ over $x, x_{\alpha}(x)=$ $\Pi_{*}\left(T_{\alpha}(v)\right)=0 \Longleftrightarrow T_{\alpha}(v) \subset \mathbf{V}_{i}$ for some $i \Longleftrightarrow v \subset \mathbf{V}_{i}$ for some $i$. The second equivalence is a consequence of (7).

Next we will show that the component functions $m_{\alpha}$ of the map $m$ can be computed using the $\alpha$-weight decomposition. This will be very useful. We use the notation in (5), (6) and (7) to state

Lemma 1.2. Suppose $\alpha \in i$ f. Suppose $v \in \mathbf{V}-\{0\}$ lies over $x \in \mathbf{P}(\mathbf{V})$

(i) $m(x)=\frac{2\left\langle v, T_{\alpha}(v)\right\rangle}{\|v\|^{2}}=\frac{2}{\|v\|^{2}} \sum_{i} a_{i}\left|v_{i}\right|^{2}$

(ii) $1 / 2 m_{\alpha}(x)$ is a convex combination of the weights $a_{i}$ such that $v_{i} \neq 0$.

(iii) $d \Pi^{*} m_{\alpha}(v)=\frac{4}{\|v\|^{2}} \operatorname{Re}\left\langle, T_{\alpha} v-\frac{m_{\alpha}(x)}{2} v\right\rangle$

(iv) $d \Pi{ }^{*} m_{\alpha}(v)=\frac{4}{\|v\|^{2}} \operatorname{Re} \sum_{i}\left(a_{i}-\frac{m_{\alpha}(x)}{2}\right)\left\langle, v_{i}\right\rangle$

(v) The gradient of $m_{\alpha}$, with respect to the Fubini-study metric on $\mathbf{P}(\mathbf{V})$ determined by the 1-1 form $\omega$, is $X_{\alpha}$. 
(vi) For $\beta \in \mathfrak{f}$, the hamiltonian vector field of $m_{i \beta}$ on $\mathbf{P}(\mathbf{V})$, is $X_{\beta}$. Thus $d m_{i \beta}=\omega\left(, X_{\beta}\right)$.

(vii) $m_{A d k^{-1}(\alpha)}(x)=m_{\alpha}(k \cdot x)$ for any $k \in K$.

Proof. Part (i) follows from (2) and (7)

$$
\begin{aligned}
m_{\alpha}(x) & =\left.\frac{d}{d t}\right|_{t=0}\|\exp t \alpha \cdot v\|^{2}=\left.\frac{d}{d t}\right|_{t=0}\left(\sum_{i} e^{2 a_{i} t}\left|v_{i}\right|^{2}\right)=2 \sum_{i} a_{i}\left|v_{i}\right|^{2} \\
& =2\left\langle v, T_{\alpha}(v)\right\rangle .
\end{aligned}
$$

Part (ii) is trivial. Parts (iii) and (iv) follow directly from (i). To deduce part (v) from part (iii), we recall the Fubini-study metric as we norm? lized it. Let $(,)_{x}$ denote the Fubini-study metric on $\mathbf{P}(\mathbf{V})$ at $x$. Identify $\mathbf{V}$ with $T_{v} \mathbf{V}$, the tangent space to $\mathbf{V}$ at $v$. For any vector $w_{i} \in \mathbf{V}$ write $w_{i}=\lambda v+w_{i}^{\frac{1}{}}$, where $\left\langle w_{i}^{\frac{1}{i}}, v\right\rangle=0$. Then

$$
\left(\Pi_{*_{\nu}} w_{1}, \Pi_{*_{\nu}} w_{2}\right)_{x}=\frac{4}{\|v\|^{2}}\left\langle w_{1}^{\frac{1}{1}}, w_{2}^{\frac{1}{2}}\right\rangle=\frac{4}{\|v\|^{2}}\left\langle w_{1}, w \frac{1}{2}\right\rangle .
$$

Also note

$$
\left\langle T_{\alpha}(v)-\frac{m_{\alpha}(x)}{2} v, v\right\rangle=0 .
$$

Thus

$$
\operatorname{grad} m_{\alpha}(x)=\Pi_{*, v}\left(T_{\alpha}(v)-\frac{m_{\alpha}(x)}{2} v\right)=\Pi_{*, v} T_{\alpha}(v)=X_{\alpha}(x) .
$$

For part (vi),

$$
\omega\left(\Pi_{*, v} w_{1}, \Pi_{*, \nu} w_{2}\right)_{x}=\frac{4}{\|v\|^{2}} \operatorname{Im}\left\langle w_{1}^{1}, w_{2}^{\frac{1}{2}}\right\rangle
$$

Suppose $\beta \in \mathfrak{f}$. Then $\operatorname{Re}\left\langle, T_{i \beta}(v)\right\rangle=\operatorname{Re}\left(-i\left\langle, T_{\beta}(v)\right\rangle\right)=$ $\operatorname{Im}\left\langle, T_{\beta}(v)\right\rangle$. Thus

$$
\frac{4}{\|v\|^{2}} \operatorname{Re}\left\langle, T_{i \beta}(v)\right\rangle=\omega\left(, \Pi_{*, v} T_{\beta}(v)\right)=\omega\left(, X_{\beta}(x)\right) .
$$


Finally, we check the $k$-equivariance in part (vii). Recall $A d(k)$ is the differential of the automorphism of $G: g \rightarrow K g K^{-1}$. Now

$$
\begin{aligned}
\|v\|^{2} \cdot m_{\alpha}(k \cdot x) & =\left.\frac{d}{d t}\right|_{t=0}\|\exp t \alpha \cdot k v\|^{2}=2 \operatorname{Re}\left\langle k \cdot v,\left.\frac{d}{d t}\right|_{t=0} \exp t \alpha \cdot k v\right\rangle \\
& =2 \operatorname{Re}\left\langle v,\left.\frac{d}{d t}\right|_{t=0}\left(k^{-1} \exp t \alpha k\right) \cdot v\right\rangle \\
& =2 \operatorname{Re}\left\langle v, T_{A d k^{-1}(\alpha)}(v)\right\rangle=\|v\|^{2} m_{A d k^{-1}(\alpha)}(x) . \quad \text { Q.E.D. }
\end{aligned}
$$

CoRollary 1.2.1. After identifying $\mathfrak{f}$ with $i \mathfrak{f}, \beta \rightarrow i \beta, m$ is a moment map.

Proof. Define $\bar{m}: \mathbf{P}(\mathbf{V}) \rightarrow \mathfrak{k}$ by $\bar{m}_{\beta}=m_{i \beta}, \beta \in \mathfrak{f}$. Then by part (vi), $d \bar{m}_{\beta}=\omega\left(, X_{\beta}\right)$. Next we must check that $\bar{m}$ is $K$-equivariant. Since $A d\left(\mathrm{k}^{-1}\right)$ is complex linear $\bar{m}_{\beta}(k \cdot x)=m_{A d k^{-1}(\beta)}(x)$ by part (vii).

It remains to see that $\bar{m}_{A d k^{-1}(\beta)}(x)=\left(A d^{*} k \cdot m\right)_{\beta}(x)$ where $A d^{*} k \cdot m$ is the coadjoint action of $K$ on $k^{*}$. But this just follows from the definition of the coadjoint action. Let $():, \mathfrak{g}^{*} \times \mathfrak{g} \rightarrow \mathbf{R}$ denote the natural pairing. Then $\left(A d^{*} k \cdot \alpha^{*}, \beta\right) \underset{\text { def }}{\bar{E}}\left(\alpha^{*}, A d k^{-1}(\beta)\right)$. Thus $\bar{m}$ is $K$-equivariant. Q.E.D.

2. Links Between the Moment Map and Geometric Invariant Theory. Let $\mathbf{V}$ be the representation of a reductive group $\mathbf{G}$ as in Section 1 . We will retain the assumptions of the first section.

The basic problem of geometric invariant theory is the construction of orbit spaces. One then wants to understand the orbit spaces in concrete cases. A fundamental theorem states that there are enough polynomial functions on $\mathbf{V}$, which are invariant under $G$, to distinguish between closed $G$-invariant sets. For characteristic 0, this was proved by Mumford in Chapter 1, Section 2 of [M]. These $G$-invariant polynomial functions are then the regular functions on the orbit space. Mumford's work on the construction of orbit spaces in [M] lead him to develop the geometric notion of a stable vector.

Definition 2.1. Suppose $v$ is a nonzero vector in $\mathbf{V}$.

(i) $v$ is unstable if the zero vector is in the closure of the orbit of $v$, $0 \in \overline{G \cdot v}$

(ii) $v$ is semistable of $0 \notin \overline{G \cdot v}$ 
(iii) $v$ is nice semistable if the orbit $G \cdot v$ is closed

(iv) $v$ is stable if the orbit $G \cdot v$ is closed and the stabilizer subgroup of $v, G_{v}$, is finite.

The $G$-invariant polynomial functions, thus, cannot distinguish the unstable vectors from the zero vector. The unstable vectors form the "null cone". The moment map will lead to a stratification of the null cone.

Notion (iii) is new; we will show in this section that it expresses a link to the moment map.

Let $\mathbf{P}(\mathbf{V})_{s s}, \mathbf{P}(\mathbf{V})_{s}, \mathbf{P}(\mathbf{v})_{u}$ denote the images via $\Pi: \mathbf{V}-\{0\} \rightarrow \mathbf{P}(\mathbf{V})$ of the sets of semistable, stable, and unstable vectors in $\mathbf{V}$, respectively. Let $\mathbf{P}(\mathbf{V})_{c}$ denote the image via $\Pi$ of the nice semistable vectors; i.e. those with closed orbits.

The first link between the moment map and geometric invariant theory is made via the notion of minimal vector, studied in [K-N].

Definition 2.2. A nonzero vector $v \in \mathrm{V}$ is a minimal vector if $d \rho_{v}(e)=$ 0 where

$$
\rho_{v}: G \rightarrow \mathbf{R} \text { is defined by } g \rightarrow\|g \cdot v\|^{2} .
$$

The image $x=\Pi(v) \in \mathbf{P}(\mathbf{V})$ of a minimal vector is a minimal point.

We next recall some results from [K-N]. Part (i) of the result justifies the definition.

Theorem 2.1. Let $\mathcal{O} \subset \mathrm{V}$ denote a G-orbit. Consider the restriction of \|\|$^{2}$ to $\Theta$ :

(i) Any critical point $v$ of $\left.\|\|^{2}\right|_{\Theta}$ is a point where $\left.\|\|^{2}\right|_{\Theta}$ attains its minimum.

(ii) $\left.\|\|^{2}\right|_{\mathcal{O}}$ attains its minimum if and only if $\Theta$ is a closed orbit, if and only if $\mathcal{O}$ consists of nice semistable vectors.

(iii) If $\Theta$ is a closed orbit, the minimum value of $\left.\|\|^{2}\right|_{\odot}$ is attained on a unique $K$ coset.

A link between the moment map and geometric invariant theory then is expressed as:

Theorem 2.2. Let $m: \mathbf{P}(\mathbf{V}) \rightarrow i i^{*}$ denote the moment map.

(i) $m^{-1}(0)$ is the set of minimal points

(ii) $G \cdot m^{-1}(0)=\mathbf{P}(\mathbf{V})_{c}$, the nice semistable points

(iii) $\mathbf{P}(\mathbf{V})_{s} \supset G \cdot m^{-1}(0)=\mathbf{P}(\mathbf{V})_{c} \supset \mathbf{P}(\mathbf{V})_{s s}$

(iv) 0 is a regular value of $m$ if and only if $\mathbf{P}(\mathbf{V})_{s}=m^{-1}(0)=\mathbf{P}(\mathbf{V})_{s s}$. 
Proof. The first three statements are easily checked. Part (i) follows immediately from the definition $m(x)=\left(d \rho_{v}(e)\right) /\|v\|^{2}, v \neq 0$ over $x$, and from the definition of minimal points as those for which $d \rho_{v}(e)=0$. Part (ii) follows from part (i) and Theorem 2.1 part (ii). Part (iii) follows immediately from (ii) and Definition 2.1. The last part (iv) of the theorem follows easily from the next three lemmas. The first was proved in [G-S].

Lemma 2.1. (Guillemin-Sternberg). For $x \in \mathbf{P}(\mathbf{V})$, the following are equivalent:

(i) $d m(x): T_{x}(\mathbf{P}(\mathbf{V})) \rightarrow i \mathfrak{f} *$ is not surjective

(ii) there exists $\alpha \in i$ such that $\operatorname{dm}_{\alpha}(x)=0$

(iii) the canonical vector field vanishes at $x, X_{\alpha}(x)=0$

(iv) the one-parameter subgroup $\exp t \alpha \subset G_{x}$, the stabilizer subgroup of $x$.

Proof of lemma. (i) $\Leftrightarrow$ (ii) is obvious. The other three parts are consequences of Lemmas 1.1. and 1.2.

Lemma 2.2. Suppose $x \in \mathbf{P}(\mathbf{V})$ and suppose $v$ lies over $x, v \neq 0$. Let $G_{x}$ and $G_{v}$ denote the subgroups of $G$ which fix $x$ and $v$ respectively.

(i) $G_{x} \supset G_{v}$

(ii) $G_{x} / G_{v} \approx \mathrm{C}^{*}$ or $G_{x} / G_{v}$ is finite

(iii) $G_{x} / G_{v} \approx C^{*} \Rightarrow v$ is unstable

(iv) v semistable $\Rightarrow G_{x} / G_{v}$ is finite, $\operatorname{dim} G_{x}=G_{v}$.

Proof of Lemma. (i) is obvious and (iv) is logically equivalent to (iii). For (ii) consider the exact sequence

$$
0 \rightarrow G_{v} \rightarrow G_{x} \rightarrow C^{*} \cdot \mathbf{V} \approx C^{*}
$$

The first homomorphism is inclusion; the second is $g \rightarrow g \cdot v$. The image of $G_{x}$ is a complex algebraic subgroup of $\mathbf{C}^{*}$, hence is either finite or all of $\mathbf{C}^{*}$. For part (iii) $G_{x} / G_{v} \approx C^{*}$ implies that there exists $\alpha \in \operatorname{Lie} G_{x} \cap i$ f such that $\exp t \alpha \cdot v=e^{a t} v$, some $a \in \mathbf{R}, a \neq 0$. We may assume $a<0$. Then $\lim _{t \rightarrow \infty}$ $\exp t \alpha \cdot v=0$, so $0 \in \overline{G \cdot v}$ and $v$ is unstable.

Lemma 2.3. Suppose $x$ is semistable, and suppose $v$ lies over $x$, $v \neq 0$. Then $\overline{G \cdot v}$ contains a unique closed orbit.

Proof of Lemma. This is a corollary of the fundamental result (Corollary $1.2[\mathrm{M}]$ ) which says that there are enough $G$-invariant polynomials to 
distinguish closed $G$-invariant sets. If $\overline{G \cdot v}$ contained more than one closed orbit, say $\mathcal{\Theta}_{1}$ and $\mathcal{\Theta}_{2}$, there would be a $G$-invariant polynomial $f, f \equiv 1$ on $\vartheta_{1}$ and $f \equiv 0$ on $\Theta_{2}$. This contradicts the fact that $f$ is constant on $\overline{G \cdot v}$. On the other hand $\overline{G \cdot v}$ contains a closed orbit-an orbit of minimal dimension. Finally, we can give the

Proof of part (iv) of the theorem. 0 is not a regular value of $m \Leftrightarrow$ there exists $x \in m^{-1}(0)$ such that $d m(x): T_{x}(\mathbf{P}(\mathbf{V})) \rightarrow i \mathfrak{i} *$ is not surjective $\Leftrightarrow$ there exists $v \in \mathbf{V}, G \cdot v$ closed and $\operatorname{dim} G_{v} \geq 1 \Leftrightarrow$ by part (iv) of 2.1 and 2.2 there exists $x \in \mathbf{P}(\mathbf{V})_{s s}-\mathbf{P}(\mathbf{V})_{s}, m(x)=0 \Leftrightarrow$ by $2.3 \mathbf{P}(\mathbf{V})_{s s}-$ $\mathbf{P}(\mathbf{V})_{\mathrm{s}} \neq \phi$. Q.D.

A canonical construction is the "Marsden-Weinstein" reduction determined by a moment map $m$. It is the set of $K$ orbits of $m^{-1}(0)$, $K \backslash m^{-1}(0)$.

In [GS], Guillemin and Sternberg proved, essentially using the notion of minimal vectors

TheOREM 2.3. Suppose $\mathbf{P}(\mathbf{V})_{s}=\mathbf{P}(\mathbf{V})_{\text {ss }}$, and suppose $G$ acts freely on $m^{-1}(0)$. Then $K \backslash m^{-1}(0)$ is a smooth complex projective variety.

The analogue in geometric invariant theory is obtained by modding out by the following equivalence relation. Suppose $v \neq 0$ lies over $x \in \mathbf{P}(\mathbf{V})$; suppose $w \neq$ lies over $y \in \mathbf{P}(\mathbf{V})$

def. $x \sim y \quad$ if $\overline{G \cdot v}$ and $\overline{G \cdot w}$ contain the same closed orbit.

By Lemma 2.3, this is an equivalence relation. We will call this "extended G-equivalence".

THEOREM 1.4 .

(i) $\mathbf{P}(\mathbf{V})_{\text {ss }}$ modulo extended G-equivalence is the Marsden-Weinstein reduction. That is, there is a set isomorphism

$$
\sim \backslash \mathbf{P}(\mathbf{V})_{s s} \approx K \backslash m^{-1}(0)
$$

(ii) $\sim \mathbf{P}(\mathbf{V})_{\text {ss }}$ is a projective algebraic variety.

Proof. For part (i) one simply notes that each set consists of one point for each closed orbit. Part (ii) follows from Guillemin and Sternberg's theorem if $G$ acts freely on $m^{-1}(0)$. In general this is Mumford's theorem 1.10 in [1].

Q.E.D. 
In this paper, we want to show that one can use the moment map approach to get some more information about $\sim \mathbf{P}(\mathbf{V})_{s s}$.

3. Another Link. Another link between the moment map and geometric invariant theory can be made because of the fundamental

Theorem 3.1. Hilbert [M]. The closure of the $G$ orbit of $v$ contains $0, \overline{G \cdot v} \ni 0$, if and only if there is an algebraic one-parameter $s u b$ group $\lambda \subset G$ such that 0 is in the closure of the $\lambda$ orbit of $v$.

This allows one to reduce questions of stability to numerical questions about the actions of one-parameter subgroups. In fact, one can do slightly better. Among one-parameter subgroups $\lambda$, there are those which are compatible with $K$ in the sense that $\lambda$ is the complexification of its intersection with $K$. In fact, one can reduce questions of stability to numerical questions about the action of one-parameter subgroups compatible with $K$. For give $\alpha \in \mathfrak{g}$ one can define a parabolic subgroup by

$$
P(\alpha)=\left\{g \in G: \lim _{t \rightarrow-\infty} g \exp t \alpha g^{-1} \text { exists in } G\right\} .
$$

In the $P(\alpha)$ conjugacy class $p \cdot \exp t \alpha p^{-1}, p \in P(\alpha)$, there is a unique representative which is given by exponentiating an element in $i f$.

Thus we will define two numerical functions for $\exp t \alpha, \alpha \in i$ f.

For $\alpha \in i$ f, denote the $\alpha$-weight decomposition of $\mathbf{V}$ by

$$
\mathbf{V}=\oplus \mathbf{V}_{i}
$$

where $\alpha$ acts on $\mathbf{V}_{i}$ by weight $a_{i}$. For $v \in \mathbf{V}$, write

$$
v=\Sigma v_{i}, \quad v_{i} \in \mathbf{V}_{i}
$$

Thus $\exp t \alpha \cdot v_{i}=e^{a_{i} t} v_{i}$. The first numerical function is defined by

$\mu(x, \alpha)=\min \left\{a_{i}: v_{i} \neq 0\right\}, \quad$ where $v$ lies over $x \quad v \neq 0, \quad x \in \mathbf{P}(\mathbf{V})$.

Thus if $\mu(x, \alpha)>0, \lim _{t \rightarrow-\infty} \exp t \cdot v=0$, for over $x$, so $x$ is unstable.

Now fix a positive definite ad- $K$ invariant inner product (, ) on $i$ f. Let $\|\alpha\|^{2}=(\alpha, \alpha)$ for $\alpha \in i f$. Define a composite numerical function 


$$
M(x)=\sup _{\substack{\alpha \in i f \\ \alpha \neq 0 .}} \frac{\mu(x, \alpha)}{\|\alpha\|}
$$

(10) Remark. Mumford proved [M] that the supremum is attained, so there exists $\alpha \in i f, M(x)=\mu(x, \alpha) /\|\alpha\|$. The first easy observation is: if $M(x)>0, x$ is unstable. The function $M$ is $G$-invariant [see $M$ or N].

The whole numerical connection is given in:

TheOREM 3.2. The Hilbert-Mumford Numerical Criterion for stability [M]

(i) $x \in \mathbf{P}(\mathbf{V})$ is unstable if and only if there exists $\alpha \in i f$ such that $\mu(x, \alpha)>0$ if and only if $M(x)>0$.

(ii) $x \in \mathbf{P}(\mathbf{V})$ is semistable, but not stable, if and only if there exists $\alpha \in i$ if such that $\mu(x, \alpha)=0$ if and only if $M(x)=0$

(iii) $x \in \mathbf{P}(\mathbf{V})$ is stable if and only if, for all $\alpha \in i \mathfrak{f}, \mu(x, \alpha)<0$-so $x$ is stable if and only if $M(x)<0$.

Now fix an ad- $K$ invariant positive definite product (, ) on $i f$.

To state a link between the moment map and geometric invariant theory, it will be useful to introduce the dual moment map

$$
m^{*}: \mathbf{P}(\mathbf{V}) \rightarrow \text { if } \quad x \rightarrow m^{*}(x), \quad \text { where } m(x)=\left(m^{*}(x),\right)
$$

The numerical functions $M: \mathbf{P}(\mathbf{V}) \rightarrow \mathbf{R}$ and $\mu(, \alpha): \mathbf{P}(\mathbf{V}) \rightarrow \mathbf{R}$ give bounds on $\|m\|(x) \overline{\overline{\text { def }}}\left\|m^{*}(x)\right\|$ and the component functions $m_{\alpha}$ of the moment map. If the bounds are attained, special phenomena occur.

LemMa 3.1. For $x \in \mathbf{P}(\mathbf{V})$ and $\alpha \in i f$

(i) $\mu(x, \alpha) \leq\left(m_{\alpha}(x)\right) / 2$

(ii) $\mu(x, \alpha)=\left(m_{\alpha}(x)\right) / 2$ if and only if $\exp t \alpha \in G_{x}$ if and only if $d m_{\alpha}(x)=0$

(iii) $\frac{\mu(x, \alpha)}{\|\alpha\|} \leq \frac{1}{2} \frac{m_{\alpha}(x)}{\|\alpha\|}<\frac{\|m\|(x)}{2}$

(iv) $M(x) \leq \frac{\|m\|(x)}{2}$

(v) $M(x)=(\|m\|(x)) / 2$ if and only if $d m_{m^{*}(x)}(x)=0$ if and only if $\exp t m^{*}(x) \in G_{x}$ 
(vi) If $M(x)=\frac{\|m\|(x)}{2}, \quad$ then $M(x)=\sup _{\alpha \in i^{\mathfrak{f}}} \frac{\mu(x, \alpha)}{\|\alpha\|}$

$$
=\frac{\mu\left(x, m^{*}(x)\right)}{\left\|m^{*}(x)\right\|} \text {. }
$$

Proof of the Lemma. To check part (i) we use Lemma 1.2(i). In terms of the $\alpha$-weight decomposition for $v$ over $x, v \neq 0$,

*

$$
\frac{m_{\alpha}(x)}{2}=\frac{\sum a_{i}\left|v_{i}\right|^{2}}{\|v\|^{2}} \geq \min \left\{a_{i}: v_{i} \neq 0\right\}=\mu(x, \alpha) .
$$

For part (ii), it is clear that equality holds in * if and only if $v$ is contained in one $\alpha$-weight space. This is equivalent to $\exp t \alpha \in G_{x}$. By Lemmas 1.1. and 1.2 this is also equivalent to $X_{\alpha}(x)=d m_{\alpha}(x)=0$. The last inequality in (iii) is easy. Just notice that

$$
\frac{m_{\alpha}(x)}{2\|\alpha\|}=\frac{1}{2}\left(m^{*}(x), \frac{\alpha}{\|\alpha\|}\right)=\frac{\left\|m^{*}(x)\right\|}{2}=\frac{\|m\|(x)}{2}
$$

Part (iv) follows from part (iii) and the definition of $M(x)$. The last two parts of (v) are equivalent by part (iv), so we need only check the first equivalence in $(v)$. For $\Leftarrow$ assume $d m_{m^{*}(x)}(x)=0$. By parts (ii) and (iii)

$$
\frac{\mu\left(x, m^{*}(x)\right)}{\|m\|(x)}=\frac{1}{2} \frac{m_{m^{*}(x)}(x)}{\|m\|(x)}=\frac{1}{2}\left(m^{*}(x), \frac{m^{*}(x)}{\|m\|(x)}\right)=\frac{1}{2}\|m\|(x)
$$

so

$$
M(x)=\sup _{\alpha \in i \mathfrak{i f}} \frac{\mu(x, \alpha)}{\|\alpha\|} \geq \frac{1}{2}\|m\|(x)
$$

Hence by (iv), $M(x)=(1 / 2)\|m\|(x)$. We will next assume part (vi) and prove $\Rightarrow$ in $(v)$. We assume, then

$$
M(x)=\frac{\|m\|(x)}{2}=\frac{\mu\left(x, m^{*}(x)\right)}{\|m\|(x)}
$$

Thus, $\mu\left(x, m^{*}(x)\right)=(1 / 2) m_{m *(x)}$ so by part (ii) $\exp t m^{*}(x) \in G_{x}$. Part (vi) follows from Remark (10). If $M(x)=(\|m\|(x)) / 2$ there exists $\alpha \in i f$ such 
that $M(x)=(\mu(x, \alpha)) /\|\alpha\|=(\|m\|(x)) / 2$. Thus by (iii) $\left(m_{\alpha}(x)\right) /\|\alpha\|=$ $\left(m^{*}(x), \alpha /\|\alpha\|\right)=\|m\|(x)$ so $\alpha=c m^{*}(x)$ for some $c \geq 0$. Q.E.D.

For special unstable points $x$, there is another connection between the moment map and geometric invariant theory.

Definition. If $x \in \mathbf{P}(\mathbf{V})$ and $M(x)=(\mu(x, \alpha)) /\|\alpha\|, \alpha \in i \mathfrak{i f}$. Then $\exp t \alpha$ is an adapted one-parameter subgroup for $x$.

Lemma 3.2. For $x \in \mathbf{P}(\mathbf{V})$, let $G_{x} \subset G$ denote the stabilizer subgroup of $x$. Assume $\exp m^{*}(x) \subset G_{x}$ and assume $m^{*}(x) \neq 0$. Then

(i) $x$ is unstable

(ii) $\exp \mathrm{tm}^{*}(x)$ is adapted for $x$.

Proof. The hypotheses and Lemma 3.1 imply that $\exp t m *(x) \cdot v)=$ $[\exp (t\|m\|(x)) / 2] v$, for $v$ over $x, v \neq 0$. Thus $\lim _{t \rightarrow-\infty} \exp t m^{*}(x) \cdot v=$ 0 , since $m^{*}(x) \neq 0$, so $x$ is unstable. The definition of adapted and Part (vi) of Lemma 3.1 imply exp $\operatorname{tm}^{*}(x)$ is adapted for $x$.

Q.E.D.

We will close this section by recalling a result of-Kempf's which gives content to the notion of adapted, for unstable points $x$.

Define $P(\alpha) \subset(G)$ to be the subgroup

$$
P(\alpha)=\left\{p \in G: \lim _{t \rightarrow-\infty} \exp t \alpha \cdot p \cdot \exp (-t \alpha) \text { exists in } G\right\} .
$$

$P(\alpha)$ is a parabolic subgroup and hence fixes a flag. Note that if $\alpha$ is adapted for $x$, so is any positive multiple of $\alpha$. Also $P(c \alpha)=P(\alpha), c>0$. Thus each ray of adapted $\alpha$ 's determines a flag.

TheOREM-KeMPF $[\mathrm{K}]$. Suppose $x \in \mathbf{P}(\mathbf{V})$ is unstable. There exists a unique ray of adapted $\alpha$ 's in if.

Thus there is a unique "worst flag" for each unstable $x$.

4. A Rationality Result for $M^{*}$. In this section we first recall that there is a natural notion of integral, and hence rational, elements in $i f$; then we prove Theorem 4.1.

We will say $\beta \in i f$ as integral if $\exp (2 \pi i \beta)=e$. This is equivalent to saying there is an algebraic one-parameter subgroup $\lambda: C^{*} \rightarrow G$ such that

$$
\exp (t \beta)=\lambda\left(e^{t}\right)
$$

Then $\beta \in i f$ is rational if $n \beta$ is integral for some $n \in \mathbf{Z}$. 
If $T \subset G$ is a torus such that $T$ is the complexification of $K \cap T$, then

$$
\text { Lie } T \approx \mathfrak{f}_{T} \oplus i \mathfrak{f}_{T} \quad \text { where } \mathfrak{f}_{T}=\operatorname{Lie}(K \cap T) \text {. }
$$

The integral elements of $i \mathfrak{f}_{T} \subset i \mathfrak{f}$ form a lattice of maximal rank in $i \mathfrak{f}_{T}$. For the set of nontrivial algebraic one-parameter subgroups of $T, \Gamma(T)$ is a free abelian group, because $T \approx \mathrm{C}^{*} \times \cdots \times \mathrm{C}^{*}(r$ times $)$ so the one parameter subgroups are

$$
\lambda(t)=\left(t^{n_{1}}, \ldots, t^{n_{r}}\right) \quad \text { for some } r \text {-tuple of integer } n_{1}, \ldots, n_{r} .
$$

Thus there is a natural isomorphism

$$
i \mathfrak{f}_{T} \approx \Gamma(T) \otimes_{\mathrm{z}} \mathbf{R} \text {. }
$$

Globally the Cartan decomposition implies

$$
i \mathfrak{f}=U_{t \in \mathfrak{i}} \mathfrak{i}_{T}
$$

where $t$ is the set of maximal tori "in $K$ ", i.e. $T \subset G$ is the complexification of $T \cap K$.

A natural condition, which we will henceforth assume, on the ad- $K$ invariant inner product is

$$
(x, y) \in \mathbf{Z} \quad \text { for all } x, y \text { integral such that }
$$

$$
[x, y]=0, \quad(\text { e.g. } x=y) .
$$

Kempf proved that each adapted ray $\mathbf{R}^{+} \alpha, \alpha$ adapted for $x$, contains an integral point.

Proposition 4.1. $\operatorname{Kempf}(K)$ for $x \in \mathbf{P}(\mathbf{V})$

(i) there exists $\alpha \in i$ if such that $M(x)=(\mu(x, \alpha)) /\|\alpha\|$

(ii) If $M(x)=(\mu(x, \alpha)) /\|\alpha\|$, the ray $\mathbf{R}^{+} \alpha$ contains an integral $\beta \in i \mathfrak{i}$.

Theorem 4.1. Let $G_{x} \subset G$ denote the stabilizer group of $x \in \mathbf{P}(\mathbf{V})$. Assume condition (12) holds. If $\exp \mathrm{tm}^{*}(x) \in G_{x}$ then $m^{*}(x)$ is rational. 
Proof. If $m^{*}(x)=0$, the theorem is trivially true, so we will assume $m^{*}(x) \neq 0$. By Lemma 3.1, $M(x)=\left[\mu\left(x, m^{*}(x)\right)\right] /(\|m\|(x))$ so by Proposition 4.1, the ray $\mathbf{R}^{+} \cdot m^{*}(x) \subset i f$ contains an integral element, $\alpha_{o}$. Let $\tilde{\alpha}_{o}=\alpha_{o} /\left\|\alpha_{o}\right\|$. Complete $\tilde{\alpha}_{o}$ to an orthonormal basis $\tilde{\alpha}_{o}, \alpha_{1}, \ldots, \alpha_{n}$ of $i$ f. Write $m^{*}(x)$ as a linear combination of these basic elements. Thus

$$
m^{*}(x)=m_{\tilde{\alpha}_{o}}(x) \tilde{\alpha}_{1}+\sum_{i=1}^{n} m_{\alpha_{i}}(x) \alpha_{i}
$$

But for $i \geq 1, m_{\alpha_{i}}(x)=\left(m^{*}(x), \alpha_{i}\right)=c\left(\tilde{\alpha}_{0}, \alpha_{1}\right)=0$ for some $c>0$. Since $m_{\tilde{\alpha}_{o}}(x)=1 /\left\|\alpha_{o}\right\|^{2} m_{\alpha_{o}}(x)$,

$$
m^{*}(x)=\frac{1}{\left\|\alpha_{o}\right\|} m_{\alpha_{o}}(x) \alpha_{o} .
$$

By condition (12), $\left\|\alpha_{o}\right\|^{2} \in \mathbf{Z}$. Also exp $t \alpha_{o} \in G_{x}$, and $\alpha_{o}$ integral imply $m_{\alpha_{o}}(x)=\mu\left(x, \alpha_{o}\right) \in \mathbf{Z}$. Thus $m^{*}(x)$ is a rational multiple of an integral element $\alpha_{o}$, so $m^{*}(x)$ is rational.

Q.E.D.

5. The Convexity Theorem. Fix a maximal torus $T \subset G$ and a Borel subgroup $T \subset B \subset G$. Recall that $i \mathfrak{f}_{t} \approx \Gamma(T) \otimes \mathbf{R}$, so $B$ determines a positive Weyl chamber $i \mathfrak{f}_{t}^{+} \subset i \mathfrak{f}_{t}$. Since $m^{*}: \mathbf{P}(\mathbf{V}) \rightarrow i \mathfrak{i f}$ is $K$ equivariant, with respect to the adjoint mapping on $i$, the image of each $K$-orbit under $m^{*}, m^{*}(k \cdot x)$ has unique point of intersection with the positive Weyl chamber. Let $\check{m}(x)=m^{*}(k \cdot x) \cap i \mathfrak{f}_{t}^{+}$, denote the unique point of intersection. Thus we have

$$
\check{m}: \mathbf{P}(\mathbf{V}) \rightarrow i \mathfrak{f}_{t}^{+} \quad x \rightarrow \check{m}(x) .
$$

TheOREM (GUILlemin-STERnBerg). The image via $\check{m}$ of a G invariant closed set in $\mathbf{P}(\mathbf{V})$ is a rational convex polytope in if ${ }^{+}$.

The theorem immediately implies

(13) Remark. Suppose $x$ is unstable then $\check{m}(G \cdot x) \nexists 0$, the distance from 0 to $\check{m}(\overline{G \cdot x}) \geq 2 M(x)$. The closest distance is attained at a unique point in $i \mathfrak{f}^{+}$.

A proof, due to Mumford, is given in an Appendix to this paper. 
6. The Norm of the Moment Map. Define the norm of the moment map by

$$
\|m\|^{2}: \mathbf{P}(\mathbf{V}) \rightarrow \mathbf{R} \quad\|m\|^{2}(x)=\left\|m^{*}(x)\right\|^{2} .
$$

Here we are using the conventions (11) and (12).

Since $m^{*}(x): \mathbf{P}(\mathbf{V}) \rightarrow i f$ is $K$-equivariant with respect to the adjoint action of $K$ on $i f$, and since \|\|$^{2}$ is ad- $K$ invariant, the norm function $\|m\|^{2}$ is constant along $K$-orbits. Thus the restriction of $\|m\|^{2}$ to $\overline{G \cdot x}$, the closure in $\mathbf{P}(\mathbf{V})$ of the $G$ orbit of $x$, may be computed from the polytope $\check{m}(\overline{G \cdot x})$

$\|m\|^{2}(x)$ is the square of the distance from the origin in $i f$ to the point $\check{m}(x)$ in the polytope $\check{m}(\overline{G \cdot x})$.

We are particularly interested in the gradient flow and the critical points of the norm function $\|m\|^{2}: \mathbf{P}(\mathbf{V}) \rightarrow \mathbf{R}$. Clearly the minimal points, where $\|m\|^{2}(x)=0$, are critical points. The other critical points we will call nonminimal critical points.

The first key fact is: at each point $x \in \mathbf{P}(\mathbf{V})$, the gradient flow of $\|m\|^{2}$ is given by the gradient flow of the (simpler) function $m_{\alpha}: \mathbf{P}(\mathbf{V}) \rightarrow \mathbf{R}$ where $\alpha=m^{*}(x)$.

Lemma 6.1. For $\alpha \in i \mathfrak{f}$, let $x_{\alpha}$ denote the canonical vector field on $\mathbf{P}(\mathbf{V})$ determined by $\alpha$

$$
\operatorname{grad}\|m\|^{2}(x)=2 X_{\alpha}(x) \quad \text { for } \alpha=m^{*}(x) .
$$

Proof. Let $\alpha_{o}, \ldots, \alpha_{n}$ denote an orthonormal basis of $i$. Then $m^{*}(y)=\Sigma_{i=0} m_{\alpha_{i}}(y) \alpha_{i}$, and $\|m\|^{2}(y)=\Sigma_{i=0}^{n} m_{\alpha_{i}}^{2}(y)$. Thus $d\|m\|^{2}(y)=2 \Sigma_{i=0} m_{\alpha_{i}}(y) d m_{\alpha_{i}}(y)$. By Lemma 1.2, part $\mathrm{v}, \operatorname{grad} m_{\alpha_{i}}=$ $x_{\alpha_{i}}$, so grad $\|m\|^{2}=\sum_{i=0}^{n} 2 m_{\alpha_{i}} x_{\alpha_{i}}$. Thus, at $x$, by linearity, $\operatorname{grad}\|m\|^{2}(x)=2 \Sigma_{i=0}^{n} m_{\alpha_{i}}(x) X_{\alpha_{i}}(x)=2 X_{m^{*}(x)}(x)$.

Recall that the numerical function $M: \mathbf{P}(\mathbf{V}) \rightarrow \mathbf{R}$ defined in (9) is $G$ invariant [N]. Using this fact and Lemma 6.1 we can characterize the critical points of the norm function.

Theorem 6.1. Suppose $x \in \mathbf{P}(\mathbf{V})$. Let $M=M(g \cdot x)$ for $g \in G$. The following four conditions are equivalent: 
(i) $x$ is a nonminimal critical point of $\|m\|^{2}: \mathbf{P}(\mathbf{V}) \rightarrow \mathbf{R}$

(ii) $\exp \operatorname{tm}^{*}(x) \subset G_{x}$, the stabilizer group of $x$ in $G$ and $m^{*}(x) \neq 0$

(iii) $M=1 / 2\left\|m^{*}(x)\right\|=1 / 2\|m\|(x)>0$

(iv) the convex polytope $\check{m}(\overline{G \cdot x})$ does not contain $0 \in i$ f ; the distance from 0 to $\check{m}(\overline{G \cdot x})$ is attained at $\check{m}(x)$ and equals $2 M$.

If $x$ is a nonminimal critical point of $\|m\|^{2}: \mathbf{P}(\mathbf{V}) \rightarrow \mathbf{R}$ then

(a) $x$ is unstable and $\exp t m^{*}(x)$ is adapted for $x$

(b) $m^{*}(x)$ is rational

COROLLARY 6.1. If $x$ and $y=g \cdot x$ are nonminimal critical points of $\|m\|^{2}: \mathbf{P}(\mathbf{V}) \rightarrow \mathbf{R}$, then $\check{m}(x)=\check{m}(y)$, and hence $\|m\|^{2}(x)=\|m\|^{2}(y)$.

Proof of the corollary. (Assuming the theorem) By part (iv) of the theorem the distance from 0 to the polytope $\check{m}(\overline{G \cdot x})=$ $\check{m}(\overline{G \cdot y})$ is attained at $\check{m}(x)$ and $\check{m}(y)$. Since the polytope is convex and does not contain 0 , the distance is attained at a unique point in $i f$. Hence $\check{m}(x)=\check{m}(y)$.

Q.E.D.

Proof of Theorem 6.1. By Lemma 6.1, $d\|m\|^{2}(x)=0$ if and only if $d m_{\alpha}(x)=0$ where $\alpha=m^{*}(x)$. Parts (ii) and (iii) are now equivalent to (i) by Lemma 3.1. Part (iv) implies $(\|m\|(x)) / 2=M>0$ which is part (iii). Next we show that (iii) implies (iv). By Remark (13), distance $(0, \check{m}(\overline{G \cdot x})) \geq 2 M$. Since $M>0,0 \notin \check{m}(\overline{G \cdot x})$. Since $\|\check{m}\|(x)=$ $\|\check{m}(x)\|=2 M$ the distance is attained at $\check{m}(x)$ and equals $2 M$. Finally, we check parts (a) and (b). By criterion (ii), $d\|m\|^{2}=0$ implies $\exp t m^{*}(x) \subset G_{x}$. Since $m^{*}(x) \neq 0$ Lemma 3.2 gives part (a) and Theorem 4.1 give parts $(b)$.

Q.E.D.

The principal result of this paper is the following theorem which generalizes parts (i) and (iii) of Theorem 2.1.

ThEOREM 6.2. LET $\left.\|m\|^{2}\right|_{G \cdot x}$ denote the restriction of $\|m\|^{2}$ : $\mathbf{P}(\mathbf{V}) \rightarrow \mathbf{R}$ to the $G$ orbit of $x \in \mathbf{P}(\mathbf{V})$. If $d\|m\|^{2}(x)=0$, then

(i) $\left.\|m\|^{2}\right|_{G \cdot x}$ attains its minimum value at $x$ **

(ii) $\left.\|m\|^{2}\right|_{G \cdot x}$ attains its minimum value on a unique $K$-coset.

Proof. If $x$ is a minimal critical point, the theorem is just Theorem 2.1 parts (i) and (iii). Hence we may assume $\|m\|^{2}(x)>0$. Part (i) is easy to check. Let $M=M(g \cdot x), g \in G$. By Lemma 3.1, $\|m\|(g \cdot x) \geq 2 M$, for

**One can check that the minimum is nondegenerate. 
all $g \in G$. By Theorem 6.1, $d\|m\|^{2}(x)=0$ implies $\|m\|(x)=2 M$. Thus $\left.\|m\|^{2}\right|_{G \cdot x}$ attains its minimum value at $x$.

To prove part (ii), it suffices to prove

*If $x$ and $y$ are critical points of $\|m\|^{2}: \mathbf{P}(\mathbf{V}) \rightarrow \mathbf{R}$ which are in the same $G$-orbit, they are in the same $K$ orbit. (This will be Theorem 7.1).

Next, we will compute the index of a critical point of $\|m\|^{2}: \mathbf{P}(\mathbf{V}) \rightarrow$ R. Denote the hessian of $\|m\|^{2}$ at a critical point $x$ by Hess $\|m\|^{2}(x)$.

Then Hess $\|m\|^{2}(x)$ is a real quadratic form on the tangent space $T_{x} \mathbf{P}(\mathbf{V})$ viewed as a real vector space. We want to compute the number of negative eigenvalues which is, by definition, the index of the critical point. This is made easier for nonminimal critical points by Theorem 6.2.

There is an orthogonal decomposition of $T_{x} \mathbf{P}(\mathbf{V})$ into the space tangent to the orbit $G \cdot x$ and the space normal to the orbit $G \cdot x$.

$$
T_{x} \mathbf{P}(\mathbf{V}) \approx T(G \cdot x) \oplus_{\mathbf{C}} N(G \cdot x) .
$$

Denote the restriction of the hessian to each of these subspaces by

$$
\begin{aligned}
& \operatorname{Hess}^{\top}\|m\|^{2}(x)=\text { Hess }\left.\|m\|^{2}(x)\right|_{T(G \cdot x)} \\
& \operatorname{Hess}^{\perp}\|m\|^{2}(x)=\text { Hess }\left.\|m\|^{2}(x)\right|_{N(G \cdot x)} .
\end{aligned}
$$

Lemma 6.2. Suppose $d\|m\|^{2}(x)=0$. Let $\alpha_{0}, \alpha_{1}, \ldots, \alpha_{n}$ denote an orthonormal basis of $i$. If $\|m\|(x) \neq 0$, we will assume $\alpha_{o}=$ $\left(m^{*}(x)\right) /(\|m\|(x))$. Then

(i) if $\|m\|(x)=0$, Hess $\|m\|^{2}(x)=2 \Sigma_{i=0}^{n} d m_{\alpha_{i}} \otimes d m_{\alpha_{i}}$.

Now assume $\|m\|(x) \neq 0$, so $x$ is a nonminimal critical point of $\|m\|^{2}$ : $\mathbf{P}(\mathbf{V}) \rightarrow \mathbf{R}$.

(ii) Hess $\|m\|^{2}(x)=2 \Sigma_{i=0}^{n} d m_{\alpha_{i}} \otimes d m_{\alpha_{i}}+\|m\|(x)$ Hess $\left.m_{\alpha_{o}}(x)\right)$. Let $\mathbf{V}=\oplus_{i \in \mathbf{t}} \mathbf{V}_{i}$ denote the weight decomposition for $m^{*}(x)$, (hence for $\alpha_{o}=\left(m^{*}(x)\right) /(\|m\|(x))$. Say $\exp \alpha_{o} t \cdot v_{i}=e^{a_{i} t} v_{i}, v_{i} \in \mathbf{V}$. Let $\mathbf{V}_{i} \approx \mathbf{V}_{i}^{\top} \oplus$ $\mathbf{V}_{i}^{\perp}$ denote the orthogonal decomposition of $\mathbf{V}_{i}$ into a space $\mathbf{V}_{i}^{\top}$ tangent to the orbit $G \cdot v$, and a space $\mathbf{V}{ }_{i}^{\perp}$ normal to the orbit $G \cdot v$, where $v$ lies over $x$, $v \neq 0$. This is possible because $\exp \left(t \alpha_{o}\right)$ stabilizes $v{ }^{*}$

\footnotetext{
*The spaces $\mathbf{V}_{i}^{\top}$ are the root spaces $\mathrm{g}_{\lambda} \subset \mathrm{g}=$ Lie $G$, with respect to the adjoint action of $\alpha_{o}$ so $\mathfrak{g}_{\lambda}=\left\{x \in \mathfrak{g} \mid\left[\alpha_{o}, x\right]=\lambda \cdot x\right\}$, and $\mathfrak{g}_{\lambda} / \mathfrak{g}_{\lambda} \cap$ Lie $G_{x} \cong \mathbf{V}_{i}^{\top}$ if $a_{i}-a_{i_{o}}=\lambda$.
} 
(iii) Some weight $a_{i_{o}}=(\|m\|(x)) / 2$

Choose $v$ over $x, w$ and $w^{\prime}$ orthogonal to $v$

(iv) Hess $\Pi^{*} m_{\alpha_{o}}(v)\left(\Pi_{* v} w^{o}, \Pi_{*_{v}} w^{\prime}\right)\left(4 /\|v\|^{2}\right) \Sigma_{i \in \mathcal{S}}\left(a_{i}-(\|m\|(x)) /\right.$ 2) $\left.\operatorname{Re}\left\langle w, w^{\prime}\right\rangle\right|_{\mathbf{v}_{i}}$, for $v$ over $x$

(v) Hess $\|m\|^{2}(x)=$ Hess $^{\top}\|m\|^{2}(x)+$ Hess $^{\perp}\|m\|^{2}(x)$

(vi) index Hess $\|m\|^{2}(x)=$ index Hess ${ }^{\perp}\|m\|^{2}(x)$.

(vii) $\operatorname{Hess}^{\perp}\|m\|^{2}(x)=2\|m\|(x)$ Hess $^{\perp} m_{\alpha_{o}}(x)$

(viii) index $\operatorname{Hess}^{\perp}\|m\|^{2}(x)=2 \quad \sum_{i \in \mathcal{S}}, \operatorname{dim}_{\mathbf{C}} \mathbf{V}^{\perp}{ }_{i}^{\perp}$.

$$
a_{i} \frac{\|m\|(x)}{2}
$$

Using the notation of the Lemma 6.2, we record the answer in

THEOREM 6.3. Suppose $x$ is a critical point of $\|m\|^{2}: \mathbf{P}(\mathbf{V}) \rightarrow \mathbf{R}$

(i) If $x$ is a minimal point, the index of $x$ is zero

(ii) Otherwise the index of $x$ is $2 \Sigma \operatorname{dim}_{\|m\|(x)} \mathbf{V}_{i}^{\perp}$

$$
a_{i}<\frac{\|m\|(x)}{2}
$$

(iii) The zero eigenspace of $\operatorname{Hess}^{\perp}\|m\|^{2}(x)$ is $\mathbf{V}_{i_{o}}$, the orthogonal complement of the line over $x$ in $\mathbf{V}_{i_{o}}^{\perp}$.

We can easily deduce the theorem from the lemma. Part (i) of the lemma implies part (i) of the theorem. Part (ii) of the theorem follows from part (vi) and (viii) of the lemma. Part (iii) of the lemma follows parts (iii), (iv) and (vii) of the lemma. Part (iii) shows that the critical point $\mathrm{x}$ can be degenerate (in directions not tangent to the $K$-orbit through $x$ ).

Proof of Lemma 6.2. Since the $\alpha_{i}$ 's form an orthonormal basis of $i$, $m^{*} y=\Sigma_{i=0} m_{\alpha_{i}}(y) \alpha_{i}, y \in \mathbf{P}(\mathbf{V})$, so $\|m\|^{2}=\Sigma_{m \alpha_{i}}^{2}$. Thus $d\|m\|^{2}=$ $\Sigma 2 m_{\alpha_{i}} d m_{\alpha_{i}}$ and hence

* Hess $\|m\|^{2}=2 \Sigma d m_{\alpha_{i}} \otimes d m_{\alpha_{i}}+2 \sum m_{\alpha_{i}}$ Hess $m_{\alpha_{i}}$.

If $m^{*}(x)=0, m_{\alpha_{i}}(x)=0$ for $i=0,1, \ldots, n$ so the second term drops out, and part (i) is verified. If $m^{*}(x) \neq 0, m_{\alpha_{o}}(x)=\|m\|(x)$ since $\alpha_{o}=$ $\left(m^{*}(x)\right) /\left\|m^{*}(x)\right\|$ and $m_{\alpha_{i}}(x)=\left(m^{*}(x), \alpha_{i}\right)=0$ for $i=1, \ldots, n$. Thus part (ii) is true. For (iii), we note that $x$ a nonminimal critical point of $\|m\|^{2}$ implies $\exp t m^{*}(x) \subset G_{x}$ which implies $v$, over $x$, is in one weight space $\mathbf{V}_{i_{o}}$. Thus 


$$
m_{\alpha_{o}}(x)=\frac{\left.\frac{d}{d t}\right|_{t=0}\left\|\exp t \alpha_{o} \cdot v\right\|^{2}}{\|v\|^{2}}=\left.\frac{d}{d t}\right|_{t=0} e^{2 a_{i_{o}}}=2 a_{i_{o}} .
$$

But $m_{\alpha_{o}}(x)=\|m\|(x)$ so $a_{i_{o}}=(\|m\|(x)) / 2$, as we wanted to show.

Assuming part (iv) for the moment, we can check the rest of the lemma. For part (v), we want to show that the subspaces $N(G \cdot x)$ and $T(G \cdot x)$ of $T_{x} \mathbf{P}(\mathbf{V})$ are orthogonal with respect to the form Hess $\|m\|^{2}(x)$. It suffices by (ii) to check that $N(G \cdot x)$ and $T(G \cdot x)$ are orthogonal for $d m_{\alpha} \otimes d m_{\alpha}, \alpha \in i f$ and for Hess $m_{\alpha_{o}}$. But kernel $d m_{\alpha}(x) \supset N(G \cdot x)$, since grad $m_{\alpha}=x_{\alpha} \in T(G \cdot x)$. Thus the subspaces are orthogonal for $d m_{\alpha} \otimes d m_{\alpha}$. Next note that $\Pi_{*}\left(\oplus \mathbf{V}_{i}^{\top}\right)=N(G \cdot x)$, and $\Pi_{*}\left(\oplus \mathbf{V}_{i}^{\top}\right)=$ $T(G \cdot x)$ where $\Pi\left(\mathbf{V}-\{0\} \rightarrow \mathbf{P}(\mathbf{V})\right.$. Since $\mathbf{V}_{i}^{\perp}$ and $\mathbf{V}_{i}^{\top}$ are orthogonal by definition, (iv) implies that $\oplus \mathbf{V}_{i}^{\top}$ and $\oplus \mathbf{V}_{i}^{\perp}$ are orthogonal with respect to Hess $\Pi * m_{\alpha_{o}}(x)$. Part (vi) follows from Theorem 6.2. The eigenvalues of Hess $^{\top}$ are 0 or positive since $x$ is minimum of $\left.\|m\|^{2}\right|_{G \cdot x}$. Thus index Hess $\|m\|^{2}(x)=$ index Hess ${ }^{\perp}\|m\|^{2}(x)$. Part (vii) follows from part (ii) and the fact that $\left.d m_{\alpha_{i}} \otimes d m_{\alpha_{i}}\right|_{N(G \cdot x} \equiv 0$ since kernel $d m_{\alpha} \supset N(G \cdot x)$. For the last part, just note that $\Pi_{*} \mid \mathbf{v}_{i}$ is an isomorphism for each $i$ such that $a_{i} \neq(\|m\|(x)) / 2$, since $v$, over $x$, is contained in $\mathbf{V}_{i_{o}}=a_{i_{o}}=$ $(\|m\|(x)) / 2$. Clearly each $\mathbf{V}_{i}$ is an eigenspace and the eigenvalue is negative only if $a_{i}-(\|m\|(x)) / 2<0$. Finally, we multiply by 2 because we are computing real dimension. To complete the proof of the lemma, we must compute the formula in part (iv).

$$
\begin{aligned}
\Pi^{*} m_{\alpha_{o}}(v)= & \left.\frac{1}{\|v\|^{2}} \frac{d}{d t}\right|_{t=0}\left\|\exp t \alpha_{o} \cdot v\right\|^{2}=\frac{1}{\|v\|^{2}}\left(\Sigma 2 a_{i}\left|v_{i}\right|^{2}\right) \\
d \Pi^{*} m_{\alpha_{o}}(v)(w)= & \frac{2}{\|v\|^{2}} \Sigma a_{i}\left(\left\langle v_{i}, w_{i}\right\rangle+\left\langle w_{i}, v_{i}\right\rangle\right) \\
& -m_{\alpha_{o}}(x) \frac{(\langle v, w\rangle+\langle w, v\rangle)}{\|v\|^{2}} \\
= & \frac{2}{\|v\|^{2}} \Sigma\left(a_{i}-\frac{m_{\alpha_{o}}(x)}{2}\right)\left(\left\langle v_{i}, w_{i}\right\rangle+\left\langle w_{i}, v_{i}\right\rangle\right) .
\end{aligned}
$$

Differentiating again, and recalling that $d m_{\alpha_{o}}(x)=0$, since $x$ exp $t \alpha_{o} \in$ $G_{x}$, and $m_{\alpha_{o}}(x)=\|m\|(x)$ gives 


$$
\begin{aligned}
\text { Hess } \Pi^{*} m_{\alpha_{o}}(v)\left(w, w^{\prime}\right)= & \frac{2}{\|v\|^{2}} \Sigma\left(a_{i}-\frac{\|m\|(x)}{2}\right)\left(\left\langle w_{i}^{\prime}, w_{i}\right\rangle+\left\langle w_{i}, w_{i}^{\prime}\right\rangle\right) \\
& + \text { a term that vanishes if }\left\langle w^{\prime}, v\right\rangle=0 .
\end{aligned}
$$

Thus

$$
\text { Hess } \begin{aligned}
m_{\alpha_{o}}(v)\left(\Pi_{* v} w, \Pi_{*_{v}} w^{\prime}\right) & =\left.\frac{4}{\|v\|^{2}} \Sigma\left(a_{i}-\frac{\|m\|(x)}{2}\right) \operatorname{Re}\left\langle w, w^{\prime}\right\rangle\right|_{v_{i}} \\
& =\frac{4}{\|v\|^{2}} \Sigma\left(a_{i}-\frac{\|m\|(x)}{2}\right) \operatorname{Re}\left\langle w_{i}, w_{i}^{\prime}\right\rangle .
\end{aligned}
$$

7. The Uniqueness of Critical Points of $\|m\|^{2}$. In this section we will complete the proof of Theorem 6.2 by proving

THEOREM 7.1. If $x$ and $y$ are nonminimal critical points of $\|m\|^{2}$ : $\mathbf{P}(\mathbf{V}) \rightarrow \mathbf{R}$ which are in the same $G$-orbit, they are in the same $K$-orbit.

Proof. We will deduce the theorem from two lemmas and then prove the two lemmas. First note the hypothesis implies, by Corollary 6.1.1, that $\|m\|^{2}(x)=\|m\|^{2}(y)>0$. Denote by $P$ the parabolic subgroup $P\left(m^{*}(x)\right)$ determined by $m^{*}(x)$, as in (12). (Since $x$ is a nonminimal critical point, $\left.m^{*}(x)=0\right)$. Let $L \subset P$ denote the Levi subgroup which centralizes $\exp t m^{*}(x)$. By the Levi decomposition $P=L \cdot U$, where $U \subset P$ is the unipotent radical of $P$. Also $G=K L U$, since $m^{*}(x) \in i$ f. Thus $y=k \ell u \cdot x$, where $k \in K, \ell \in L, u \in U$. Thus $y=k \ell u \cdot x$, where $k \in K, \ell \in L, u \in U$. Since $y$ is a critical point of $\|m\|^{2}$ if and only if the $K$ orbit $K y$ consists of critical points of $\|m\|^{2}$, it suffices to prove the theorem for $y=\ell u \cdot x$. Using this notation we state

LeMma 7.1. Suppose $x$ and $y=\ell u \cdot x$ are nonminimal critical points of $\|m\|^{2}$. Then $u=e$, the identity in $G$.

Lemma 7.2. Suppose $\beta \in i$ f, $\exp t \beta \notin G_{x}$. Then $m_{\beta}(\exp t \beta \cdot x)$ is $a$ strictly increasing function of $t$.

Assuming the lemmas, we will deduce the theorem. By Lemma 7.1, $y=\ell \cdot x$. If $\ell \in G_{x}, y=x$ and we are done. Otherwise $y=\ell \cdot x, \ell \notin G_{x}$. We will show that $\ell \notin G_{x}$ implies $\|m\|^{2}(y)>\|m\|^{2}(x)$. This contradiction will prove the theorem. Let $K_{L}=K \cap L$. Since $K_{L} \backslash L$ is a symmetric space, there is a geodesic joining the cosets $[e]$ and $[\ell]$. Thus there is a real one- 
parameter subgroup $\exp t \alpha, \alpha \in i \mathfrak{i}_{L}$ such that the orbit $[\exp t \alpha \cdot e]$ contains $[\ell]$. Hence $\exp t_{o} \alpha \cdot k_{1} x=k_{2} y$ for some $k_{1}, k_{2} \in \mathbf{R}$. Note that $m^{*}\left(k_{1} \cdot x\right)=A d k_{1} \cdot m^{*}(x)$, since $k_{1} \in L$, which centralizes exp $\mathrm{tm}^{*}(x)$. Now replace $x$ by $k_{1} \cdot x$ and $y$ by $k_{2} \cdot y$, so $\exp t_{o} \alpha \cdot x=y$. We may assume $\alpha$ is of length 1 and perpendicular to $\alpha_{o}=\left(m^{*}(x)\right) /(\|m\|(x))$ since $\exp t^{*}(x) \subset G_{x}$. We may also assume $t_{o}>0$. Set $\alpha=\alpha_{1}$ and set $\|m\|^{2}(t)=\|m\|^{2}\left(\exp t \alpha_{1} \cdot x\right)$. Complete $\alpha_{0}, \alpha_{1}$ to an orthonormal basis of $i$ f. Set $m_{\alpha_{i}}\left(\exp t \alpha_{1} \cdot x\right)=m_{\alpha_{i}}(t)$. Then

$$
\|m\|^{2}(t)=\sum_{i=0}^{n} m_{\alpha_{i}}^{2}(t) \geq m_{\alpha_{0}}^{2}(t)+m_{\alpha_{1}}^{2}(t) .
$$

Now $m_{\alpha_{0}}(t)$ is constant since $\exp t \alpha_{1}$ commutes with $\exp s \alpha_{0}$ and $\exp s \alpha_{0} \epsilon$ $G_{x}$. For, if $v$ lies over $x, v \neq 0$, then

$$
\begin{gathered}
m_{\alpha_{0}}(t)=\left.\frac{1}{\left\|\exp t \alpha_{1} \cdot v\right\|^{2}} \frac{d}{d s}\right|_{s=0}\left\|\exp s \alpha_{0} \cdot \exp t \alpha_{1} \cdot v\right\|^{2} \text { so } \\
m_{\alpha_{0}}(t)=\left.\frac{1}{\left\|\exp t \alpha_{1} \cdot v\right\|^{2}} \frac{d}{d s}\right|_{s=0}\left\|e^{s a_{i_{o}}} \exp t \alpha_{1} \cdot v\right\|^{2}=2 a_{i_{o}} \\
\text { where } 2 a_{i_{o}}=\|m\|(x) .
\end{gathered}
$$

Thus $\|m\|^{2}(t) \geq\|m\|^{2}(x)+m_{\alpha_{1}}^{2}(t)$.

But by Lemma 7.2, $m_{\alpha_{1}}(t)$ is strictly increasing. Since $m_{\alpha_{1}}(0)=0$ and $t_{o}>0, m_{\alpha_{1}}^{2}\left(t_{o}\right)>0$. We conclude

$$
\|m\|^{2}(y)=\|m\|^{2}\left(t_{o}\right)>\|m\|^{2}(0)=\|m\|^{2}(x) .
$$

This contradicts the fact that $\|m\|^{2}(x)=\|m\|^{2}(y)$. Hence $\ell=e$, and $y=$ $x$, so the theorem follows from the lemmas.

Now we prove the lemmas. The first lemma follows from the wellknown

General Lemma. Suppose $\alpha \in i$ if. Let $U(\alpha)$ denote the unipotent radical of the parabolic $P(\alpha)$ associated to $\exp t \alpha$. Let $\mathbf{V}=\oplus \mathbf{V}_{i}$ denote the $\alpha$-weight decomposition of $\mathbf{V}$; say $\exp t \alpha \cdot v_{i}=e^{t a_{i}} v_{i}$ for $v_{i} \in \mathbf{V}_{i}$. Then for $v_{i} \in \mathbf{V}_{i}, u \in U$

$$
u \cdot v_{i}-v_{i} \in \underset{a_{j}>a_{i}}{\oplus} \mathbf{V}_{j} \text { and } u \cdot v_{i}-v_{i} \neq 0
$$


Proof of Lemma 7.1. We will show $u \neq e$ implies $\|m\|^{2}(y)>$ $\|m\|^{2}(x)$. This contradiction will prove the lemma. Let $\mathbf{V}=\oplus \mathbf{V}_{i}$ denote the $m^{*}(x)$ weight decomposition of $\mathbf{V}$. This is also the weight decomposition for $\alpha_{o}=\left(m^{*}(x)\right) /(\|m\|(x))$. Say exp $t \alpha_{0} \cdot v_{i}=e^{t a_{i}} v_{i}, v_{i} \in \mathbf{V}_{i}$. Suppose $v \in \mathbf{V}, v \neq 0$ lies over $x$. Then $v \in \mathbf{V}_{i_{o}}$. from $i_{o}$, since $\exp t m^{*}(x) \subset G_{x}$. Since $u$ is in the unipotent radical of $P=P\left(m^{*}(x)\right)$, the general lemma implies

$$
u \cdot v-v=\sum_{a_{j}>a_{i_{o}}} v_{j} \quad \text { where some } v_{j} \neq 0
$$

or $u \cdot v=\Sigma_{a_{j} \geq a_{i_{j}}} v_{j}, v_{i_{o}}=v$, some $v_{j} \neq 0 a_{j}>a_{i_{o}}$. Since $\ell$ commutes with $\exp t \alpha_{o}, \ell v_{j} \in \mathbf{V}_{j}$, so the $\alpha_{o}$-weight decomposition of $\ell u \cdot v$ is

$$
\ell u \cdot v=\sum_{a_{j} \geq a_{i_{o}}} \ell v_{j} .
$$

Thus by Lemma 1.2,

$$
m_{\alpha_{o}}(\ell u \cdot x)=\frac{1}{\|\ell \cdot v\|} \sum_{a_{j} \geq a_{i_{o}}} a_{j}\left\|\ell_{j}\right\|^{2} .
$$

Since some $v_{j} \neq 0, a_{j}>a_{i_{o}}$.

$$
m_{\alpha}(\ell u \cdot x)>2 a_{i_{o}}=m_{\alpha}(x)=\|m\|(x)>0 .
$$

Since $\|m\|^{2}(y)>m_{\alpha_{o}}^{2}(y)=m_{\alpha_{o}}^{2}(\ell u \cdot x)$, we have shown $u \neq e$ implies

$$
\|m\|^{2}(y)>\|m\|^{2}(x) .
$$

This is a contradiction, so $u$ must be $e$, and Lemma 7.1 is proved.

Proof of Lemma 7.2. We will show $(d / d t) m_{\beta}(\exp t \beta \cdot x)>0$. Let $\mathbf{V}=\oplus \mathbf{V}_{i}$ denote the weight decomposition of $\mathbf{V}$ for $\exp t \beta$; say $\exp t \beta v_{i}=$ $e^{t b_{i}} v_{i}$ for $v_{i} \in \mathbf{V}_{i}$ for $v \in \mathbf{V}$ over $x, v \neq 0$, write $v=\Sigma v_{i}, v_{i} \in \mathbf{V}_{i}$. Then

$$
\exp t \beta \cdot v=\Sigma e^{t b_{i}} v_{i}
$$

By Lemma 1.2 part (i)

$$
m_{\beta}(t) \underset{\text { notation }}{=} m_{\beta}(\exp t \beta \cdot x)=\frac{\Sigma 2 b_{i} e^{2 t b_{i}}\left|v_{i}\right|^{2}}{\Sigma e^{2 t b_{i}}\left|v_{i}\right|^{2}} .
$$


If one computes the derivative one finds

$$
\frac{d}{d t} m_{\beta}(t)=\frac{4 \sum_{i<j}\left(b_{i}-b_{j}\right)^{2} e^{2 t\left(b_{i}+b_{j}\right)}\left|v_{i}\right|^{2}\left|v_{j}\right|^{2}}{\left(\sum e^{2 t b_{i}}\left|v_{i}\right|^{2}\right)^{2}} .
$$

This expression is not identically zero since $\exp t \beta \notin G_{x}$ implies there is more than one nonzero term in the sum $\exp t \beta \cdot v=\Sigma e^{t b_{i}} v_{i}$, and if $i \neq j$, $b_{i} \neq b_{j}$. Hence

$$
\frac{d}{d t} m_{\beta}(t)>0
$$

Thus Lemma 7.2 is verified and so the proof of Theorem 7.1 is complete.

Q.E.D.

8. A General Example: Weight Vectors Determine Critical Points of $\|\boldsymbol{m}\|^{2}$. Let $T \subset G$ denote a maximal torus such that $T$ is the complexification of $K \cap T$. Let $\mathbf{V}=\oplus \mathbf{V}_{x}$ denote the $T$-weight decomposition of $\mathbf{V}$, so $T$ acts on $V_{x}$ by the character $\chi$. There is a perfect pairing between the characters of $T, \chi(T)$, and the nontrivial algebraic one-parameter subgroups of $T, \Gamma(T)$.

$$
\chi(T) \times \Gamma(T) \rightarrow \mathbf{Z} \quad(x, \tau) \rightarrow\langle x, \tau\rangle .
$$

This clearly extends to a nondegenerate pairing $\chi(T) \otimes \mathbf{R} \times \Gamma(T) \otimes$ $\mathbf{R} \rightarrow \mathbf{R}$. Since $\Gamma(T) \otimes \mathbf{R} \approx i \mathfrak{f}_{T}^{*}, \chi(T) \otimes \mathbf{R} \approx i \mathfrak{f}_{T}$. There is an obvious inclusion.

$$
\chi(T) \otimes \mathbf{R} \rightarrow i \mathfrak{l} *
$$

Namely $\langle x, \alpha\rangle=0$ for each $\alpha \in i \mathfrak{i f}$ orthogonal to $i f_{T}$.

Proposition 8.1. Suppose $y_{\chi} \in \mathbf{P}(\mathbf{V})$ is the image of a nonzero weight vector $v_{\chi} \in \mathbf{V}_{\chi}$. Then

(i) $m\left(y_{\chi}\right)=2 \chi$

(ii) $y_{\chi}$ is a critical point of $\|m\|^{2}: \mathbf{P}(\mathbf{V}) \rightarrow \mathbf{R}$

(iii) index $\left(y_{\chi}\right) \geq 2 \sum_{\substack{\left(\chi^{\prime}, \chi\right)=\|\chi\|^{2}, \chi^{\prime} \neq \chi \\ \chi^{\prime} \neq \chi+\rho, \rho \text { a root for } G .}} \operatorname{dim}_{\mathbf{C}} \mathbf{V}_{\chi^{\prime}}$ 
In (iii), the inner product $\left(\chi^{\prime}, \chi\right)$ is the inner product induced on $i \mathfrak{f}^{*} \approx$ $\chi(T) \otimes \mathbf{R}$ by (,) on if.

Proof. For (i) we first note that if $\alpha \in i f_{t}$, then

$$
m_{\alpha}\left(y_{\chi}\right)=\frac{\left.\frac{d}{d t}\right|_{t=0}\left\|\exp t \alpha \cdot v_{\chi}\right\|^{2}}{\left\|v_{\chi}\right\|^{2}}=\left.\frac{d}{d t}\right|_{t=0} e^{2(\chi, \alpha) t}=2\langle\chi, \alpha\rangle .
$$

Thus we need only show that if $\alpha \in i \mathfrak{f}_{T}^{\perp}$, the orthogonal complement of $i f_{T}$ in $i f$, then $m_{\alpha}\left(y_{\chi}\right)=0$. One can choose a basis $\alpha_{i}$ of $i \mathfrak{f}_{\top}^{\perp}$ such that $\alpha_{i}=$ $\beta_{i}+\gamma_{i}$ where $\beta_{i}$ and $\gamma_{i}$ in Lie $G$ are each root vectors. Denote the roots of $G$ associated with $\beta_{i}$ and $\gamma_{i}$ respectively by $\rho_{\beta_{i}}$ and $\rho_{\gamma_{i}}$. Recall the

General Fact. Suppose $\rho$ is a root for G. Suppose $\beta \in$ Lie $G$ is in the $\rho$-root space. If $v \in \mathbf{V}_{\chi}$ is a $T$-weight vector, then

$$
\left.\frac{d}{d t}\right|_{t=0} \exp t \beta \cdot v \in \mathbf{V}_{\chi+\rho}
$$

so

$$
\begin{aligned}
m_{\beta}(y) & \left.\overline{\overline{\text { def }}} \frac{1}{\|v\|^{2}} \frac{d}{d t}\right|_{t=0}\|\exp t \beta \cdot v\|^{2} \\
& =\frac{1}{\|v\|^{2}} 2 \operatorname{Re}\left\langle v,\left.\frac{d}{d t}\right|_{t=0} \exp t \beta \cdot v\right\rangle=0 .
\end{aligned}
$$

Then this general fact implies $m_{\alpha_{i}}\left(y_{\chi}\right)=m_{\beta_{i}}\left(y_{\chi}\right)+m_{\gamma_{i}}\left(y_{\chi}\right)=0+0=0$ so kernel $m_{\alpha} \supset i \mathfrak{f}_{T}^{\perp}$. Hence part (i) of the proposition is verified. Part (ii) now follows easily from part (i). For by part (i) $\exp t m^{*}\left(y_{\chi}\right) \subset T \subset G_{y_{\chi}}$, so $y_{\chi}$ is a critical point by Theorem 6.1. For part (iii) we compute the index using the formula in Theorem 6.3. There is an orthogonal decomposition $\mathbf{V}=N\left(G \cdot v_{\chi}\right) \oplus T\left(G \cdot v_{\chi}\right)$ into the spaces normal and tangent to the orbit $G \cdot v_{\chi}$ respectively. Let $\mathbf{V}_{\chi^{\prime}}^{\perp}=\mathbf{V}_{\chi^{\prime}} \cap N\left(G \cdot v_{\chi}\right)$. Then by the formula

$$
\operatorname{index}\left(y_{\chi}\right)=2 \underset{\left(x, x^{\prime}\right)<\|x\|^{\|^{\prime}}}{\sum} \operatorname{dim}\left(\mathbf{V}_{\chi^{\prime}}^{\perp}\right) \text {. }
$$

But if $\chi^{\prime} \neq \chi+\rho$ for any root $\rho$ for $G, \mathbf{V}_{\chi^{\prime}}=\mathbf{V}_{\chi^{\prime}}^{\perp}$, so the estimate in part (iii) is true.

Q.E.D. 
9. The Stratification of the Null Cone and the Limit Set. The gradient flow of $\|m\|^{2}$ determines a stratification of the null cone. Each point $x \in N \subset \mathbf{P}(\mathbf{V})$ is carried via the gradient flow into a critical point of $\|m\|^{2}$. Each $K$-orbit $\langle\alpha\rangle=K \cdot \alpha \subset$ if determines a stratum-namely the set of points $N_{\langle\alpha\rangle} \subset N$ which flow into a critical point $x$ such that $m^{*}(x) \in$ $K \cdot \alpha=\langle\alpha\rangle$. Our computation $\operatorname{grad}\|m\|^{2}(x)=2 X_{\alpha}(x)$, where $\alpha=$ $m^{*}(x)$ and $X_{\alpha}$ is the canonical vector field determined by $\alpha$, shows that the trajectories of the gradient flow are tangent to the $G$-orbits. The limit set $C_{\langle\alpha\rangle}$ of the gradient flow in the stratum $N_{\langle\alpha\rangle}$ is the set of critical points $x$ of $\|m\|^{2}$ with $m^{*}(x) \in\langle\alpha\rangle$. The limit set $C_{\langle\alpha\rangle}$ is $K$-invariant. The first result of the section is

THEOREM 9.1. $C_{\langle\alpha\rangle / K}$ is a projective variety.

To prove the theorem, we will have to produce a projective variety whose points are in one-to-one correspondence with the $K$-orbits of critical points $C_{\langle\alpha\rangle}$, in a natural way. Recall that a $G$-orbit contains at most one $K$-orbit of critical points of $\|m\|^{2}$, by Theorem 6.2 ., so the theorem could also be stated as: $G \cdot C_{\langle\alpha\rangle / G}$ is a projective variety.

We will first define a smaller set $C_{\alpha} \subset C_{\langle\alpha\rangle}$ and a "smaller" group $K^{\prime}$, which depends on $\alpha$, such that there is a set bijection $C_{\langle\alpha\rangle / K} \approx C_{\alpha / K^{\prime}}$. We will then prove that $C_{\alpha / K^{\prime}}$ is naturally a projective variety. Define

$$
C_{\alpha}=\left\{x: d\|m\|^{2}=0 \text { and } m^{*}(x)=\alpha\right\} \text { so } C_{\alpha} \subset C_{\langle\alpha\rangle}
$$

We will call $C_{\alpha}$ a blade of the limit set $C_{\langle\alpha\rangle}$.

Set

$$
M=\frac{\|\alpha\|}{2}
$$

Then $C_{\alpha}$ non-empty implies $\exp t(\alpha) /\|a\| \cdot v=C^{M t} v$, for $v \neq 0$, over $x \in C_{\alpha}$ by Lemma 3.1, part v. Let $\mathbf{V}_{M} \subset \mathbf{V}$ denote the $M$-weight space for $\exp t(\alpha) /\|\alpha\|$. Thus

$$
C_{\alpha} \subset \mathbf{P}\left(\mathbf{V}_{M}\right)
$$

If $C_{\alpha}$ is non-empty, then $\alpha$ is rational by Theorem 4.1 and Lemma 3.1. Thus, there exists a positive integer $n$, and an algebraic one-parameter subgroup $\tau \in \Gamma(G)$, such that $\tau \circ e^{t}=\exp n \alpha t$. Identify $\tau$ with its image in $G$ and define 


$$
\begin{gathered}
L(\tau)=\text { the centralizer of } \tau \\
L^{\prime}=L(\tau)_{/ \tau}
\end{gathered}
$$

Clearly $L^{\prime}$ is a reductive group, which is independent of the choice of $n$. Also $\mathbf{P}\left(\mathbf{V}_{M}\right)$ is $L(\tau)$ invariant and the action of $L(\alpha)$ on $\mathbf{P}\left(\mathbf{V}_{M}\right)$ induces an action of $L^{\prime}$ on $\mathbf{P}\left(\mathbf{V}_{M}\right)$ because $\tau$ fixes each $x \in \mathbf{P}\left(\mathbf{V}_{M}\right)$. Every $v \neq 0$ over $x$ in $\mathbf{P}\left(\mathbf{V}_{M}\right)$ is unstable for the action of $L(\alpha)$, since $\exp (\alpha /\|\alpha\|) t \cdot v=e^{M t} v$, so we are forced to pass to $L^{\prime}$. Let $K_{L}=K \cap L(\tau)$; let $K^{\prime} \subset L^{\prime}$ denote the image of $K_{L}$; thus $K_{L}$ and $K^{\prime}$ are maximal compact subgroups of $L(\tau)$ and $L^{\prime}$ respectively.

\section{Remark 9.1.}

(i) $C_{\langle\alpha\rangle}$ is the disjoint union of orbits of $\operatorname{cosets} K_{/ K_{L}} \cdot C_{\alpha}$

(ii) There are bijections of sets

$$
C_{\langle\alpha\rangle / K} \approx C_{\alpha / K_{L}} \approx C_{\alpha / K^{\prime}}
$$

\section{THEOREM 9.2.}

(i) $C_{\alpha}$ is the set of minimal points in $\mathbf{P}\left(\mathbf{V}_{M}\right)$ for the action of $L^{\prime}$

(ii) $C_{\langle\alpha\rangle} \subset L^{\prime} \cdot C_{\alpha} \subset \mathbf{P}\left(\mathbf{V}_{M}\right)_{s s}$, where $\mathbf{P}\left(\mathbf{V}_{M}\right)_{s s} \subset \mathbf{P}\left(\mathbf{V}_{M}\right)$ is the set of $L^{\prime}$ semistable points

(iii) $C_{\alpha_{/ K}}$ is the Marsden-Weinstein reduction of $\mathbf{P}\left(\mathbf{V}_{M}\right)$ for the moment map $m_{L^{\prime}}$ determined by $L^{\prime}$, and hence is a projective variety

(iv) $C_{\alpha_{/ K^{\prime}}} \approx \mathbf{P}\left(\mathbf{V}_{M}\right)_{s s / \sim}$ where $\sim$ is extended $L^{\prime}$ equivalence on $\mathbf{P}\left(\mathbf{V}_{M}\right)_{s s}$

COROLlary 9.2.1.

$$
C_{\langle\alpha\rangle / K} \approx C_{\alpha / K^{\prime}} \approx \mathbf{P}\left(\mathbf{V}_{M}\right)_{s s / \sim} .
$$

Hence $C_{\langle\alpha\rangle / K}$ is a projective variety. The corollary follows immediately from the remark and Theorem 9.2, and is just Theorem 9.1.

Proof of Theorem 9.2. Parts (ii), (iii) and (iv) follow from part (i) by Theorem 2.4. Thus it suffices to prove part (i). We have to show $m_{L^{\prime}}(x)=$ $0 \Leftrightarrow x \in C_{\alpha}$. Let $m_{L(\tau)}: \mathbf{P}\left(\mathbf{V}_{M}\right) \rightarrow i \mathfrak{f}_{L}$ denote the moment map for $L(\tau)$. The first observation is

$$
m=m_{L(\tau)} \quad \text { on } \mathbf{P}\left(\mathbf{V}_{M}\right)
$$


Let $U \subset P(\tau)$ denote the unipotent radical of the parabolic subgroup $P(\tau)$. Let $U^{-} \subset G$ denote the unipotent radical of the opposite parabolic subgroup. Then the Levi decomposition implies

$$
\mathfrak{g}=\operatorname{Lie}(G)=\operatorname{Lie}(L(\tau)) \oplus \operatorname{Lie}(U) \oplus \operatorname{Lie}\left(U^{-}\right)
$$

View $m(X) \in g^{*}$. It suffices to show $m(x)(\eta)=0$ for $\eta \in U$ or $\eta \in U^{-}$. However, then

$$
m_{\eta}(x)=m(x)(\eta)=\frac{2}{\|v\|^{2}}\left\langle\frac{d}{d t} \exp t \eta \cdot v, v\right\rangle=0
$$

for $v$ over $x \in \mathbf{P}\left(\mathbf{V}_{M}\right)$ by the General Lemma cited in the proof of Lemma 7.2. Hence * is checked.

Next note that there is a subgroup $L^{\prime}(\tau) \subset L(\tau)$ such that $L^{\prime}(\tau) \cap$ $\operatorname{Im} \tau$ is discrete and such that multiplication $L^{\prime}(\tau) \times \operatorname{Im} \tau \rightarrow L(\tau)$ gives a finite surjective homomorphism of groups. Thus, $\operatorname{Lie} L^{\prime}(\tau) \approx \operatorname{Lie} L(\tau)$ and Lie $\operatorname{Im} \tau=C \cdot \alpha$. Hence, we may identify the moment maps $m_{L^{\prime}}$ with $m_{L^{\prime}(\tau)}$ and $m_{\operatorname{Im} \tau}$ with $m_{\alpha}$,

$$
m=m_{L(\tau)}=m_{L^{\prime}}+m_{\alpha} \quad \text { on } \mathbf{P}\left(\mathbf{V}_{M}\right)
$$

so

$$
m_{L^{\prime}}(x)=0 \Leftrightarrow m(x)=m_{\alpha}(x)
$$

Since $x \in \mathbf{P}\left(\mathbf{V}_{M}\right) \exp t(\alpha /\|\alpha\|) \cdot v=e^{t M} v$, for $v \neq 0$ over $x$.

Lemma 3.1 implies for $x \in \mathbf{P}\left(\mathbf{V}_{M}\right)$

$$
m_{L^{\prime}}(x)=0 \Leftrightarrow m(x)=m_{\alpha}(x) \Leftrightarrow x \in C_{\alpha}
$$

Thus $C_{\alpha}$ is the set of minimal points for the action of $L^{\prime}$ on $\mathbf{P}\left(\mathbf{V}_{M}\right)$ and part (i) is proved.

Q.E.D.

In [H], Hesselink, using the notion of an adapted one-parameter subgroup, defined an algebraic stratification of the null cone. A stratum is

$$
\begin{aligned}
N_{M,\langle\tau\rangle}=\{x \in N: M(x)=M \text { and there exists a } g \in G \text { such that } & \left.x \in g^{-1} \text { is adapted for } x\right\}
\end{aligned}
$$


Since the numerical function $M: \mathbf{P}(\mathbf{V}) \rightarrow \mathbf{R}$ is $G$-invariant, the strata are $G$-invariant. Hesselink showed that the strata are Zariski-locally closed, irreducible, nonsingular, rational projective varieties.

We will study the limit set $L$ for Hesselink's stratification. We will show that the essential "pieces" of $L$ are $\mathbf{P}\left(\mathbf{V}_{M}\right)_{s s}$. Hence the quotients of the two limit sets are isomorphic projective varieties $\mathbf{P}\left(\mathbf{V}_{M}\right) s s_{/ L^{\prime}} \approx C_{\alpha_{/ K^{\prime}}}$. The relation of the two limit sets, then, will perfectly reflect the symplectogeometric definition of one and the algebro-geometric definition of the other.

The natural limit set to consider from Hesselink's stratification is

$$
L=\left\{y \in N: y=\lim _{t \rightarrow 0} \tau(t) \cdot x, \quad \tau \text { adapted for } x\right\}
$$

Let $L_{M,\langle\tau\rangle}=\left\{y=\lim _{t \rightarrow 0} \tau(t) \cdot x, x \in N_{M, \tau}\right.$ and $\tau$ adapted for $\left.x\right\}$.

*Theorem 9.3. Suppose $x \in N \subset \mathbf{P}(\mathbf{V})$ and $\tau$ is adapted for $x$. Let $y=\lim _{t \rightarrow 0} \tau(t) \cdot x$. Then

(i) $\tau$ is adapted for $y$

(ii) $M(y)=M(x)$

(iii) $\tau$ fixes $y$, so $\tau(t) \cdot y=y$ for all $t \in e^{*}$.

Corollary. $L_{M,\langle\tau\rangle}=N_{M,\langle\tau\rangle} \cap L$.

We first deduce the corollary from the theorem. The theorem implies $L_{M,\langle\tau\rangle} \subset N_{M,\langle\tau\rangle}$. Suppose $y \in L \cap N_{M,\langle\tau\rangle}$. Then $y=\lim _{t \rightarrow 0} \tau^{\prime}(t) \cdot x, \tau^{\prime}$ adapted for $x$. By the theorem, $\tau^{\prime}$ is adapted for $y$ so $\tau^{\prime} \in\langle\tau\rangle$ and $M=$ $M(y)=M(x)$. Thus $x \in N_{M,\langle\tau\rangle}$ and hence, $y \in L_{M,\langle\tau\rangle}$.

Proof of Theorem 9.3. Part (ii) follows from part (i) and the definition of adapted. For part (iii), suppose $v \in \mathbf{V}-\{0\}$ lies over $y$. The definition of $y$ implies that $v$ is in one $\tau$-weight space, so $\tau(t) \cdot=t^{n} v$. Hence, $\tau(t) \cdot y=y$. Thus, we need only check part (i). We shall first show that it suffices to prove part (i) when $G$ is a torus $T$ and then we will prove part (i) for a torus.

Suppose $\zeta \in \Gamma(G)$ is adapted for $y$. The intersection $P(\zeta) \cap P(\tau)$ contains a maximal torus $T$. Since any torus is conjugate to a subtorus of $T$, there exist $p \in P(\tau)$ and $q \in P(\zeta)$ such that $p \tau p^{-1}=\tau^{\prime}$ and $q \zeta q^{-1}=\zeta^{\prime}$ are in $\Gamma(T)$. Then $\tau^{\prime}$ is adapted for $p \cdot x=x^{\prime}$ and $\lim _{t \rightarrow 0} \tau^{\prime}(t) \cdot x^{\prime}=$ $p \cdot y=y^{\prime}$, and $y=p^{-1} y^{\prime}$.

*Ramanan and Ramanathan have recently proved part (i). 
Assume now that the theorem is proved for $T$. Set $P=P(\tau)=P\left(\tau^{\prime}\right)$. Then we could deduce that $\tau^{\prime}$ was $T$-adapted for $y^{\prime}$. However, the parabolic invariance property of $\mu$ implies that the adapted one-parameter subgroups for the $P$ orbit of $y^{\prime}$ are $P \cdot \tau^{\prime}=\left\{\tilde{p} \tau^{\prime} \tilde{p}^{-1}: \tilde{p} \in P\right\}$. Since $y=p^{-1} y^{\prime}$ and $\tau=p^{-1} \tau^{\prime} p^{-1}, \tau$ is $T$-adapted for $y$. So

$$
\mu\left(y, \frac{\tau}{\|\tau\|}\right)=\sup _{\tilde{\tau} \in \Gamma(T)} \mu\left(y, \frac{\tilde{\tau}}{\|\tilde{\tau}\|}\right)=M_{T}(y) .
$$

Since $\Gamma(T)$ contains an adapted one-parameter subgroup for $y, M_{T}(y)=$ $M(y)$. Hence, $\tau$ is adapted for $y$.

To complete the proof, we must prove part (i) for $G=T$ a torus. Let $\kappa(T)$ denote the set of characters of $T$. There is a perfect pairing $\langle$,$\rangle :$ $\kappa(T) \times \Gamma(T) \rightarrow \mathbf{Z}$. Let $\mathbf{V}=\otimes_{\chi \in \kappa(T)} \mathbf{V}_{\chi}$ denote the $T$-weight decomposition of $\mathbf{V}$, so $\tau(t) \cdot \Sigma v_{\chi}=\Sigma t^{\langle\chi, \tau\rangle} v_{\chi}$. For $x \in \mathbf{P}(\mathbf{V}), v \in \mathbf{V}-\{0\}$ over $x$, let $S_{T}(x)=\left\{\chi: v_{\chi} \neq 0\right.$ where $\left.\mathbf{v}=\Sigma v_{\chi}\right\}$. Let $\overline{S_{T}(x)} \subset \kappa(T) \otimes \mathbf{R}$ denote the convex hull. We may identify $\Gamma(T) \otimes \mathbf{R}$ with $\kappa(T) \otimes \mathbf{R}$ via the perfect pairing. Since $\mu(x,(\tilde{\tau} /\|\tilde{\tau}\|))=\min \left\{\langle x,(\tilde{\tau} /\|\tilde{\tau}\|)\rangle: \chi \in S_{T}(x)\right\}=$ the distance from 0 to the projection of $\overline{S_{T}(x)}$ onto the direction $\tilde{\tau}$,

$$
M_{T}(x)=\sup _{\tilde{\tau} \in \Gamma(T)} \mu\left(x, \frac{\tilde{\tau}}{\|\tilde{\tau}\|}\right)=\operatorname{distance}\left(0, \overline{S_{T}(x)}\right)
$$

By hypothesis $M_{T}(x)=\mu(x,(\tau,\|\tau\|))$ so the distance is attained in the direction $\tau$. By definition of $y, \overline{S_{T}(y)} \subset \overline{S_{T}(x)}$ is the closest face to 0 . Thus, $\operatorname{dist}\left(0, \overline{S_{T}(y)}\right)=\operatorname{dist}\left(0, \overline{S_{T}(x)}\right.$ and the distances are attained in the same direction $\tau$. This proves part (i) for the case of a torus, and completes the proof of the theorem.

Q.E.D.

Let $N_{M, \tau}=\left\{x \in N_{M,\langle\tau\rangle}: \tau\right.$ is adapted for $\left.x\right\}$. Hesselink called these sets blades. Let $L_{M, \tau}=N_{M, \tau} \cap L$. We will call $L_{M, \tau}$ a blade of the limit set $L \cap N_{M,\langle\tau\rangle}$. As before, let $\mathbf{V}_{M} \subset \mathbf{V}$ denote the $M$-weight space for the action of $(1 /\|\tau\|) \cdot \tau$. If $N_{M, \tau}$ is non-empty, $\mathbf{V}_{M}$ is non-empty since

$$
L_{M, \tau} \subset \mathbf{P}\left(\mathbf{V}_{M}\right)
$$

Again, as before, let $L(\tau)$ denote the centralizer of $\tau$ and let $L^{\prime}=L(\tau)_{/ \operatorname{Im} \tau}$.

THeOREM 9.4. Let $\mathbf{P}\left(\mathbf{V}_{M}\right)_{\text {ss }}$ denote the set of $L^{\prime}$ semistable points. Suppose $x \in \mathbf{P}\left(\mathbf{V}_{M}\right)$ 
(i) $x \in \mathbf{P}\left(\mathbf{V}_{M}\right)_{s s} \Leftrightarrow \tau$ is G-adapted for $x$

(ii) $\mathbf{P}\left(\mathbf{V}_{M}\right)_{s s}=L_{M, \tau}$

Note: Part (ii) follows immediately from part (i). The forward implication in (i) shows $\mathbf{P}\left(\mathbf{V}_{M}\right)_{s s} \subset L_{M, \tau}$. The backward implication in (i) shows $L_{M, \tau} \subset \mathbf{P}\left(\mathbf{V}_{M}\right)_{s s}$.

The main result of the section will follow easily from Theorem 9.2 and Theorem 9.4.

Given a rational $\alpha \in i$ ff, let $n_{\alpha}$ denote the smallest positive integer such that $n_{\alpha} \alpha$ is integral. Then there exists $\tau_{\alpha} \in \Gamma(G)$ such that $\tau_{\alpha} o e^{t}=$ $\exp n_{\alpha} \alpha t$. Let $L^{\prime}=L\left(\tau_{\alpha}\right)_{\operatorname{Im} \tau_{\alpha}}$ and let $K^{\prime} \subset L^{\prime}$ denote the image in $L^{\prime}$ of $K \cap L\left(\tau_{\alpha}\right)$, so $K^{\prime} \subset L^{\prime}$ is a maximal compact subgroup.

Using all of this previous notation, the main result is

THEOREM 9.5.

(i) There is a one-to-one correspondence between the gradient flow stratification and Hesselink's stratification* given by

$$
N_{\langle\alpha\rangle} \rightarrow N_{M,\left\langle\tau_{\alpha}\right\rangle} \quad \text { where } M=\frac{\|\alpha\|}{z}
$$

Corresponding blades of the two limit sets are related by:

(ii) $C_{\alpha} \subset L^{\prime} \cdot C_{\alpha} \subset L_{M, \tau_{\alpha}}=\mathbf{P}\left(\mathbf{V}_{M}\right)_{s s}$

(iii) $C_{\alpha_{/ K}}$ is the Marsden-Weinstein reduction of $\mathbf{P}\left(\mathbf{V}_{M}\right)$ by $K^{\prime}$

(iv) $L_{M, \tau_{\alpha / L}}$ is Mumford's quotient of $\mathbf{P}\left(\mathbf{V}_{M}\right)_{s s}$ by $L^{\prime}$

Hence

(v) There is a natural isomorphism of projective varieties

$$
C_{\alpha / K^{\prime}} \approx L_{M, \tau_{\alpha / L^{\prime}}}
$$

(vi) The quotient by $K$ of a stratum of the critical locus of $\|m\|^{2}$, $C_{\langle\alpha\rangle / K}$, is naturally isomorphic to the quotient of a blade of either of the two limit sets.

$$
C_{\langle\alpha\rangle / K} \approx C_{\alpha / K^{\prime}} \approx L_{M, \tau_{\alpha / L^{\prime}}}
$$

*F. Kirwan has proved the two stratifications are the same. However, the limit sets are not the same. 
Proof of Theorem 9.5 (Assuming Theorem 9.4). Theorem $9.4 \mathrm{im}$ plies $L_{M, \tau_{\alpha}}=\mathbf{P}\left(\mathbf{V}_{M}\right)_{s s}$. Thus, parts ii, iii, iv, v, and vi follow from Theorem 9.2 and its corollary. Hence, it suffices to check part (i). If $N_{\langle\alpha\rangle}$ is nonempty, then $C_{\alpha}$ is non-empty, so by part (ii), $N_{M,\left\langle\tau_{\alpha}\right\rangle}$ is non-empty, so the mapping is well-defined. Next we check that the mapping is injective. Suppose $\left\langle\tau_{\alpha}\right\rangle=\left\langle\tau_{\alpha^{\prime}}\right\rangle$ and $\|\alpha\|=\left\|\alpha^{\prime}\right\|=M / 2$. Then $\tau_{\alpha^{\prime}}=g \tau_{\alpha} g^{-1}$. However, $\tau_{\alpha}$ and $\tau_{\alpha^{\prime}}$ are both real, so $g \in K$, and then $k \tau_{\alpha} k^{-1}=\tau_{k \cdot \alpha}$. This implies $\alpha^{\prime}=k \cdot \alpha$ or $\langle\alpha\rangle=\left\langle\alpha^{\prime}\right\rangle$ and hence $N_{\langle\alpha\rangle}=N_{\left\langle\alpha^{\prime}\right\rangle}$. Finally we must check that the mapping is surjective. Assume $N_{M,\langle\tau\rangle}$ is non-empty. We may assume that $\tau$ is the generator of the ray in $\Gamma(G)$ which contains $\tau$. Then $\langle\tau\rangle=\left\{g n \cdot \tau g^{-1} ; n \in \mathbf{Z}^{+}, \mathrm{g} \in G\right\}$. Hence, $\langle\tau\rangle$ contains a (unique) $K$-conjugacy class of real one-parameter subgroups, so we may assume $\tau$ is real. Then $N_{M,\langle\tau\rangle}=G \cdot N_{M, \tau}$ non-empty implies $N_{M, \tau}$ non-empty which implies $L_{M, \tau}=\mathbf{P}\left(\mathbf{V}_{M}\right)_{s s}$ is non-empty. Thus $\mathbf{P}\left(\mathbf{V}_{M}\right)_{s s}$ contains a non-empty set of $L^{\prime}$ closed orbits and hence $\mathbf{P}\left(\mathbf{V}_{M}\right)_{s s}$ contains a non-empty set of $K^{\prime}$ minimal vectors. By Theorem 9.2. part i, this set is $C_{\alpha}$, where $\|\alpha\|=M / 2$ and $n \cdot \tau=\tau_{\alpha}$ for some $n \in \mathbf{Z}^{+}$. Thus $\left\langle\tau_{\alpha}\right\rangle=\langle\tau\rangle$, and $N_{\langle\alpha\rangle} \supset C_{\alpha}$ is nonempty so $N_{\langle\alpha\rangle} \rightarrow N_{M,\langle\tau\rangle}$. We have verified surjectivity.

Q.E.D.

Before proving Theorem 9.4, we will make precise the notion of semistable and unstable for the action of $L^{\prime}=L(\tau)_{/ \mathrm{Im} \tau}$.

Remark 9.1. There exists a reductive subgroup $H \subset L(\tau)$ such that $H \times C \rightarrow L(\tau)(h, t \mapsto h \cdot \tau(t)$ is a finite surjective homomorphism of groups. Thus $\Gamma\left(L^{\prime}\right) \approx \Gamma(H)$. Hence $x$ is $L^{\prime}$-semistable or $L^{\prime}$-unstable if and only if $x$ is $H^{\prime}$-semistable or $H^{\prime}$-unstable.

Lemma 9.1. Suppose $\tau(t) \cdot x=x$. Set $x^{\prime}=p \cdot x, p \in P(\tau)$; set $\tau^{\prime}=$ $p \tau p^{-1}$, so $\tau^{\prime}(t) \cdot x^{\prime}=x^{\prime}$. Let $L^{\prime \prime}=L\left(\tau^{\prime}\right)_{/\left(\operatorname{Im} \tau^{\prime}\right)}$. Then $x^{\prime}$ is $L^{\prime \prime}$ semistable if and only if $x^{\prime}$ is $L^{\prime}$ semistable.

Proof. Set $H^{\prime}=p H p^{-1}$. Since $L\left(\tau^{\prime}\right)=p L(\tau) p^{-1}$, the remark implies $x^{\prime}$ is $L^{\prime \prime}$ semistable if and only if $x^{\prime}$ is $H^{\prime}$ semistable. Suppose $v \in \mathbf{V}$ lies over $x, v \neq 0$, so $V^{\prime}=p \cdot v$ lies over $x^{\prime}=p \cdot x$. Then $0 \in \overline{H \cdot v} \Leftrightarrow 0 \in$ $\overline{H^{\prime} \cdot v^{\prime}}=\overline{p H p^{-1} \cdot p v}=p \cdot \overline{H v}$.

Q.E.D.

LemMA 9.2. Suppose $\tau(t) \cdot x=x$ and $\mu(x,(\tau /\|\tau\|)=M$. Then $x$ is $L^{\prime}=L(\tau)_{/ \operatorname{Im} \tau}$ unstable if and only if there exists $\zeta \in \Gamma(L(\tau))$ such that $\mu(x,(\zeta /\|\zeta\|))>M$.

Proof. Remark 9.1 implies $\Gamma(L(\tau)) \approx \Gamma(H) \otimes \mathbf{Z} \cdot \tau$ where $n \cdot \tau(t)=\tau\left(t^{n}\right)$ if $n \geq 0$ and $n \cdot \tau(t)=\tau^{-1}\left(t^{|n|}\right)$ for $n<0$. Given $\zeta \in$ 
$\Gamma(L(\tau))$, then there exist $\zeta^{\prime} \in \Gamma(H)$ and $n \in \mathbf{Z}$, such that $\zeta(t)=$ $\zeta^{\prime}(t)(n \cdot \tau(t))$. Clearly $\|\zeta\|>|n|\|\tau\|$ so

$$
\begin{aligned}
\mu\left(x, \frac{\zeta}{\|\zeta\|}\right) & =\mu\left(x, \frac{\zeta^{\prime}}{\|\zeta\|}\right)+n \mu\left(x, \frac{\tau}{\|\zeta\|}\right) \leq \mu\left(x, \frac{\zeta^{\prime}}{\|\zeta\|}\right)+\mu\left(x, \frac{\tau}{\|\tau\|}\right) \\
& =\mu\left(x, \frac{\zeta^{\prime}}{\|\zeta\|}\right)+M .
\end{aligned}
$$

Thus, $M<\mu(x,(\zeta /\|\zeta\|)) \Rightarrow 0<\mu\left(x, \zeta^{\prime}\right)$. Conversely

$$
0<\mu\left(x, \zeta^{\prime}\right) \Rightarrow M<\mu\left(x, \frac{\zeta^{\prime} \cdot \tau}{\left\|\zeta^{\prime} \cdot \tau\right\|}\right) .
$$

Using these Lemmas, it will be much easier to give the

Proof of Theorem 9.4. It suffices to prove part (i). Suppose $\tau$ is $G$ adapted for $x$; then the definition of adapted and the definition of $\mathbf{V}_{M}$ imply

$$
M=\mu\left(x, \frac{\tau}{\|\tau\|}\right)=M(x) \underset{\text { def. }}{\bar{\tau}} \sup _{\tau \in M(G)} \frac{\mu(x, \tau)}{\|\tau\|} .
$$

Thus Lemma 9.2 implies $x$ is not $L^{\prime}$ unstable; this is equivalent to saying that $x$ is $L^{\prime}$ semistable.

Suppose $x \in \mathbf{P}\left(\mathbf{V}_{M}\right)$ is semistable for the action of $L^{\prime}=L(\tau)_{/ \operatorname{Im} \tau}$. Since $\mathbf{V}_{M}$ is the $M$-weight space for the action of $(1 /\|\tau\|) \tau, \tau(t) \cdot x=x$ and $\mu(x,(\tau /\|\tau\|))=M$. In particular, if $v \in \mathbf{V}_{M}, v \neq 0$, lies over $x$, then $\tau(t) \cdot v=t^{a} \cdot v$, where $a=\|\tau\| \cdot M>0$, so $v$ is $G$-unstable. Hence we may seek a $G$-adapted one-parameter subgroup for $v$. We want to prove that $\tau$ is $G$-adapted for $x$, so we must show that

$$
M=\mu\left(x, \frac{\tau}{\|\tau\|}\right)=\sup _{\zeta \in \Gamma(G)} \mu\left(x, \frac{\zeta}{\|\zeta\|}\right)=M(x) .
$$

Suppose $\zeta \in \Gamma(G)$ is $G$-adapted for $x$,

Since the intersection of any two parabolic subgroups contains a maximal torus, there is a maximal torus $T \subset P(\tau) \cap P(\zeta)$. Since any torus in either $P(\tau)$ or $P(\zeta)$ is conjugate to a subtorus of $T$, there exist $p \in P(\tau)$ and $q \in$ 
$P(\zeta)$ such that $p \tau p^{-1} \underset{\text { not. }}{\overline{\tau^{\prime}} \text { and } q \zeta q^{-1}} \overline{\overline{\text { not }}} \zeta^{\prime}$ are both in $\Gamma(T)$. Then $\tau^{\prime}$ is also adapted for $x$, so $\mu\left(x,\left(\zeta^{\prime} /\left\|\zeta^{\prime}\right\|\right)\right)=M(x)>M$. Also $\zeta^{\prime} \in L\left(\tau^{\prime}\right)$, the centralizer of $\tau^{\prime}$. Let $x^{\prime}=p \cdot x$; then $\tau^{\prime}$ fixes $x^{\prime}$ and $x^{\prime}$ is semistable for the action of $L\left(\tau^{\prime}\right)_{/ \operatorname{Im} \tau^{\prime}}=L^{\prime \prime}$ by Lemma 9.1.

Claim. If

$$
\mu\left(x, \frac{\zeta^{\prime}}{\left\|\zeta^{\prime}\right\|}\right)>M, \text { then } \mu\left(x, \frac{\zeta^{\prime}}{\left\|\zeta^{\prime}\right\|}\right)>M
$$

The claim implies by Lemma 9.2, that $x^{\prime}$ is $L^{\prime \prime}$ unstable. This contradicts the fact that $x^{\prime}$ is $L^{\prime \prime}$ semistable. Thus $\mu\left(x,\left(\zeta^{\prime} /\left\|\zeta^{\prime}\right\|\right)\right) \leq M$. Since $\mu\left(x,\left(\zeta^{\prime} /\left\|\zeta^{\prime}\right\|\right)\right)=M(x)$ and $M(x) \geq M$, we conclude $M(x)=M$ and hence, $\tau$ is $G$-adapted for $x$. Thus it suffices to give the

Proof of the Claim. Let $\mathbf{V}=\otimes_{\chi \in \kappa(T)} \mathbf{V}_{\chi}$ denote the $T$-weight decomposition of $\mathbf{V}$, where $\kappa(T)$ is the set of characters of $T$. Denote the natural perfect pairing $\kappa(T) \times \Gamma(T) \rightarrow \mathbf{Z}$ by $\langle$,$\rangle . The symmetric \mathbf{Z}$ bilinear form on $\Gamma(T)$ induces, via the perfect pairing, a symmetric $\mathbf{R}$ bilinear form on $\kappa(T) \otimes_{\mathbf{z}} \mathbf{R}$. Given $y \in \mathbf{V}$, $v$ over $y, v=\Sigma v_{\chi} \neq 0, v_{\chi} \in \mathbf{V}_{\chi}$, let $S_{T}(y)=\{\chi$ : $\left.v_{\chi} \neq 0\right\}$, and let $\overline{S_{T}(y)}=$ the convex hull of $S_{T}(y)$. Then

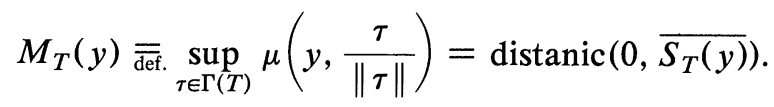

In our situation $\mu(x,(\tau /\|\tau\|))=\mu\left(x^{\prime},\left(\tau^{\prime} /\left\|\tau^{\prime}\right\|\right)\right)=M$. Since $\tau^{\prime}$ also fixes $x^{\prime}, \overline{S_{T}\left(x^{\prime}\right)}$ is contained in the hyperplane $\left\langle,\left(\tau^{\prime} /\left\|\tau^{\prime}\right\|\right)\right\rangle=M$. Since $x=$ $p^{-1} \cdot x^{\prime}, p^{-1} \in P\left(\tau^{\prime}\right), \overline{S_{T}(x)}$ is contained in the half-space $\left\langle,\left(\tau^{\prime} /\left\|\tau^{\prime}\right\|\right)\right\rangle \geq$ $M$ and $\overline{S_{T}\left(x^{\prime}\right)}$ is a face of $\overline{S_{T}(x)}$. However, $M(x)=\mu\left(x,\left(\zeta^{\prime} /\left\|\zeta^{\prime}\right\|\right)\right)=$ $M_{T}(x)=$ distance $\left(0, \overline{S_{T}(x)}\right)>M$ implies $\overline{S_{T}(x)}$ is contained in the open half space $\left\langle,\left(\zeta^{\prime} /\left\|\zeta^{\prime}\right\|\right)\right\rangle>M$. Since $\overline{S_{T}\left(x^{\prime}\right)} \subset \overline{S_{T}(x)}, \overline{S_{T}(x)}$ is also contained in the open half space $\left\langle,\left(\zeta^{\prime} /\left\|\zeta^{\prime}\right\|\right)\right\rangle>M$, which is equivalent to saying $\mu\left(x^{\prime},\left(\zeta^{\prime} /\left\|\zeta^{\prime}\right\|\right)\right)>M$. This proves the claim and completes the proof of Theorem 9.4.

Q.E.D.

10. An Example: SL $(n, C)$ Acting on Homogeneous Polynomials. In this section we will compute the moment map and some of the critical points for $\|m\|^{2}$ for the case

$$
G=\operatorname{SL}(n, \mathbf{C}) \quad K=\operatorname{SU}(n) \quad \text { and } \quad \mathbf{V}=\operatorname{Sym}^{d}\left(\mathbf{C}^{n}\right)^{*}
$$


For several cases, we can compute the minimal vectors and all of the critical points. Here the action of $G$ on $\mathbf{V}$, the vector space of homogeneous polynomials of degree $d$ in $n$ variables is just linear change of coordinates. We will let $G$ act on the right so the action is

$$
\mathbf{V} \times G \rightarrow \mathbf{V} \quad(f, g) \rightarrow f \circ g .
$$

In this case $\mathbf{P}(\mathbf{V})$ parametrizes hypersurfaces of degree $d$ in $\mathbf{P}^{n-1}$.

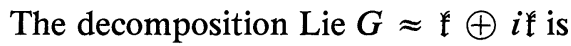

$$
\begin{aligned}
& \mathfrak{g} \ell(n)=n \times n \text { matrices }=n \times n \text { matrices }+n \times n \text { matrices }=i \mathfrak{H} \oplus \mathcal{H} \text {. } \\
& \text { trace } 0 \text { skew hermitian hermitian } \\
& \text { trace } 0 \quad \text { trace } 0
\end{aligned}
$$

An ad- $K$ invariant inner product on $\mathfrak{g} \ell(n)$ is defined by

$$
(\alpha, \beta)=\operatorname{Re}(\operatorname{trace} \alpha \beta) \quad \alpha, \beta \in \mathrm{g} \ell(n)=\operatorname{Lie}(G) \text {. }
$$

If $\alpha$ and $\beta$ are integral, $(\alpha, \beta) \in \mathbf{Z}$. Also (, ) is positive definite on $\mathcal{H C}=i \mathfrak{f}$, and $i \mathfrak{H C} \oplus \mathfrak{H C}$ is an orthogonal decomposition of $\mathfrak{g} \ell(n)$.

Thus the dual moment map $m^{*}: \mathbf{P}(\mathbf{V}) \rightarrow i f$ will assign to each hypersurface of degree $d$ in $\mathbf{P}^{n-1}$ an $n \times n$ hermitian matrix of trace 0 . The $K$ equivariance of the moment map implies that after moving the hypersurface by some unitary transformation, the assigned matrix $m^{*}\left(\left[f \circ \boldsymbol{k}^{-1}\right]\right)$ is diagonal, with real entries on the diagonal. The map $\check{m}: \mathbf{P}(\mathbf{V}) \rightarrow i \mathfrak{f}_{+}^{+}$, which maps $G$-invariant closed sets to polytopes, is equivalent to the eigenvalue map

$$
\begin{aligned}
& \lambda: \mathbf{P}(\mathbf{V}) \rightarrow \mathbf{R}^{n} \quad \lambda \rightarrow \lambda(x)=\left(\lambda_{1}(x), \ldots, \lambda_{n}(x)\right) . \\
& \text { where } \lambda_{1}(x) \geq \cdots \geq \lambda_{n}(x) \text { are the eigenvalues of } m^{*}(x) .
\end{aligned}
$$

To compute $m^{*}(x)$, we must first fix a $K$-invariant Hermitian inner product on $\mathbf{V}$. Since $\mathbf{V} \times G \rightarrow \mathbf{V}$ is an irreducible representation here, there is a unique (up to scalar multiple) $K$-invariant Hermitian inner product on $\mathbf{V}$. Let $\operatorname{Sym}\left(\otimes^{d}\left(\mathbf{C}^{n}\right)^{*}\right) \subset \otimes^{d}\left(\mathbf{C}^{n^{*}}\right)$ denote the subspace of $d$-fold tensors invariant under permutation of the factors. The induced inner product on $\otimes^{d}\left(\mathbf{C}^{n^{*}}\right)$ is $K$-invariant. Define a $K$-invariant hermitian inner product $\langle$, $\rangle$ on $\operatorname{Sym}^{d}\left(\mathbf{C}^{n^{*}}\right)$ by declaring the natural quotient map

$$
\operatorname{Sym}\left(\otimes^{d} \mathbf{C}^{n^{*}}\right) \rightarrow \operatorname{Sym}^{d}\left(\mathbf{C}^{n^{*}}\right)
$$

to be an isometry. Then the inner product $\langle$,$\rangle is computed by:$ 
(1) If $X_{1}, \ldots, X_{n}$ form an orthonormal basis of $\mathbf{C}^{n^{*}}$ the monomials of degree $d, m^{\alpha}=X_{1}^{\alpha_{1}} \cdots X_{n}^{\alpha_{n}}$ form an orthogonal basis of $\mathbf{V}=$ $\operatorname{Sym}^{d}\left(\mathbf{C}^{n^{*}}\right)$.

(2) $\left\|m^{\alpha}\right\|=\frac{1}{\left[\begin{array}{l}d \\ \alpha\end{array}\right]} \quad$ where $\left(\begin{array}{l}d \\ \alpha\end{array}\right)=\frac{d !}{\alpha_{1} ! \cdots \alpha_{n} !}$.

We will use the convention that $\langle$,$\rangle is complex linear in the first factor and$ conjugate linear in the second factor.

Now we can compute the moment map. Fix an orthonormal bases $x_{1}, \ldots, x_{n}$ of $\mathbf{C}^{n^{*}}$. Choose a defining equation $f=0$ for $x \in \mathbf{P}(\mathbf{V})$. Write $f=\Sigma c_{\alpha} m^{\alpha}, c_{\alpha} \in \mathbf{C}$. Define an $n \times n$ hermitian matrix $H(f)$ by

$$
H(f)_{i}^{j}=\frac{1}{\|f\|^{2}}\left\langle f, x_{j} \frac{\partial}{\partial x_{i}} f\right\rangle=\frac{1}{\|f\|^{2}} \frac{1}{d}\left\langle\frac{\partial f}{\partial x_{j}}, \frac{\partial f}{\partial x_{i}}\right\rangle .
$$

The second equality is an easy calculation. Clearly any two defining equations $f=0, f^{\prime}=0$ for $x$ determine the same matrix, since $f^{\prime}=\lambda f$ for some $\lambda \in \mathbf{C}-\{0\}$.

LEMma 10.1. For $\mathbf{V}=\operatorname{Sym}^{d}\left(\mathbf{C}^{n^{*}}\right), G=\operatorname{SL}(n, \mathbf{C})$, the value of the dual moment map

$$
m: \mathbf{P}(\mathbf{V}) \rightarrow i \mathfrak{f}
$$

at a hypersurface $x$ defined by $f=0$ is

$$
\frac{1}{2} m^{*}(x)=H(f)-\frac{d}{n} I
$$

where $I$ is the $n \times n$ identity matrix.

Proof. We first check that $m^{*}(x)$ is hermitian of trace 0 . The second equality in (15) shows $H(f)$ and hence $m^{*}(x)$ is hermitian. Next: trace $m^{*}(x)=2(\operatorname{trace} H(f)-d)$. But by $(15)$

$$
\operatorname{trace} H(f)=\frac{1}{\|f\|^{2}}\left\langle f, \sum_{i=1}^{n} x_{i} \frac{\partial f}{\partial x_{i}}\right\rangle=\frac{1}{\|f\|^{2}}\langle f, d f\rangle=d
$$


by Euler's formula, so trace $m^{*}(x)=0$. Next we check that the formula is correct. It suffices to check

$$
\left.\frac{1}{2} \frac{d}{d t}\right|_{t=0}\|f \circ \exp t \alpha\|^{2}=\frac{1}{2}\left(m^{*}(x), \alpha\right)
$$

for $\alpha$ running through a (real) basis of $\operatorname{Lie} \operatorname{SL}(n, \mathbf{C})$. We will use the basis $E_{i}^{j}, \sqrt{-1} E_{i}^{j}$, for $i \neq j, 1 \leq i, j \leq n$ and $E_{i}^{i}-E_{i+1}^{i+1}, \mathrm{i}=1, \ldots, n-1$. As usual $E_{i}^{j}$ is the matrix with 1 in the $i, j$ th position and zeroes elsewhere. Thus let $\alpha=E_{i}^{j}$. Then

$$
\left.\frac{d}{d t}\right|_{t=0} f \circ \exp t E_{i}^{j}=\left.\frac{d}{d t}\right|_{t=0} f\left(I+t E_{i}^{j}\right) X=x_{j} \frac{\partial f}{\partial x_{i}}
$$

so

$$
\left.\frac{1}{2} \frac{1}{\|f\|^{2}} \frac{d}{d t}\right|_{t=0}\left\|f \circ \exp t E_{i}^{j}\right\|=\frac{1}{\|f\|^{2}} \operatorname{Re}\left\langle f ; x_{j} \frac{\partial f}{\partial x_{i}}\right\rangle .
$$

Also,

$$
\frac{\operatorname{Re}}{2}\left(\operatorname{trace} m^{*}(x) E_{i}^{j}\right)=\frac{1}{2} \operatorname{Re} m^{*}(x)_{j}^{i}=\frac{1}{\|f\|^{2}} \operatorname{Re}\left\langle f, x_{i} \frac{\partial f}{\partial x_{j}}\right\rangle-\delta_{i}^{j} \frac{d}{n}
$$

Since $H(f)$ is hermitian we conclude

$$
\frac{1}{2}\left(m^{*}(x), E_{i}^{j}\right)=\frac{1}{\|f\|^{2}} \operatorname{Re}\left\langle f, x_{j} \frac{\partial f}{\partial x_{i}}\right\rangle-\delta_{i}^{j} \frac{d}{n} .
$$

Thus ** holds for $\alpha=E_{i}^{j} i \neq j$ and for $\alpha=E_{i}^{i}-E_{i+1}^{i+1}$. Next set $\alpha=$ $\sqrt{-1} E_{i}^{j}$. Then

$$
\begin{aligned}
\left.\frac{1}{2\|f\|^{2}} \frac{d}{d t}\right|_{t=0}\left\|f \circ \exp t \sqrt{-1} E_{i}^{j}\right\|^{2} & =\frac{1}{\|f\|^{2}} \operatorname{Re}\left\langle f, \sqrt{-1} x_{j} \frac{\partial f}{\partial x_{i}}\right\rangle \\
& =\frac{1}{\|f\|^{2}} \operatorname{Im}\left\langle f, x_{j} \frac{\partial f}{\partial x_{i}}\right\rangle .
\end{aligned}
$$


Since

$$
\begin{aligned}
\frac{1}{2}\left(m^{*}(x), \sqrt{-1} E_{i}^{j}\right) & =\frac{1}{2} \operatorname{Re} \sqrt{-1} m^{*}(x)_{j}^{i}=\frac{-1}{\|f\|^{2}} \operatorname{Im}\left\langle f, x_{i} \frac{\partial f}{\partial x_{j}}\right\rangle \\
& =\frac{1}{\|f\|^{2}} \operatorname{Im}\left\langle f, x_{j} \frac{\partial f}{\partial x_{i}}\right\rangle .
\end{aligned}
$$

We have checked $* *$ for $\alpha=\sqrt{-1} E_{i}^{j}$.

Q.E.D

Henceforth, for convenience, we will write

$$
m^{*}[f] \underset{\overline{\text { def }}}{\overline{\bar{n}}} m^{*}([f]), \quad \text { when } f \neq 0, f \in \mathbf{V} .
$$

It would be nice to understand which polynomials $f$ had diagonal moment matrices $m^{*}[f]$. Then, essentially, $\check{m}[f]$ is $m^{*}[f]$ up to a permutation of the diagonal elements. Also, if $m^{*}[f]$ is diagonal, then $f$ has some sort of optimal form. If

$$
H(f)=\left[\begin{array}{ccc}
h_{1}^{1} & & 0 \\
& \ddots & \\
0 & & h_{n}^{n}
\end{array}\right] \quad \text { so } m^{*}[f]=\left[\begin{array}{ccc}
h_{1}^{1}-d / n & & 0 \\
& \ddots & \\
0 & & h_{n}^{n}-d / n
\end{array}\right]
$$

We will write (when it is clear that $m^{*}[f]$ is diagonal)

$$
\mathrm{m} *[f]=h-(d / n) .
$$

If $m^{*}[f]$ is diagonal, it is easy to "graph". Recall that the convex hull of the weights $\alpha, \Sigma \alpha_{i}=d$, is a polytope in $\mathbf{R}^{n}$ with vertices " $x_{i}^{d}$ " and with barycenter $(d / n)=(d / n, \ldots, d / n)$. If $m^{*}[f]=h-(d / n)$ is diagonal, then $m^{*}[f]$ is graphed as the vector from the barycenter to $h=$ $\left(h_{1}^{1}, \ldots, h_{n}^{n}\right)$ in $\mathbf{R}^{n}$. For $n=3$, with $X_{1}=X, X_{2}=Y, X_{3}=Z$

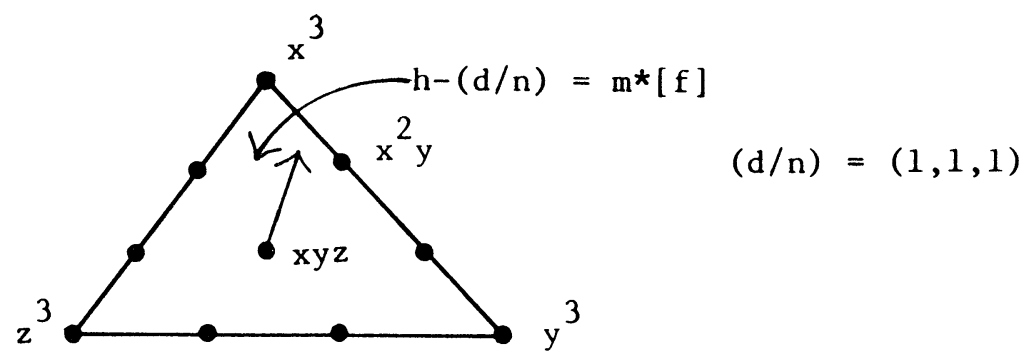


Furthermore, if $m^{*}[f]$ is diagonal, $\|m\|[f]=\|h-(d / n)\|$ where the right side is just computed from the standard Euclidean norm. With this interpretation and notation in mind, we note the following easy but graphic consequence of Lemma 10.1.

COROLlary 10.1.1**

(i) For monomials $m^{\alpha}=x_{1}^{\alpha_{1}}, \ldots, x_{n}^{\alpha_{n}}, m^{*}\left[m^{\alpha}\right]$ is diagonal and

$$
m^{*}\left[m^{\alpha}\right]=\alpha-(d / n) .
$$

(ii) If $m^{*}[f]$ is diagonal, and $f=\Sigma c_{\alpha}\left(m^{\alpha} /\left\|m^{\alpha}\right\|\right)$, then

$$
m^{*}[f]=\sum_{\alpha} \frac{\left|c_{\alpha}\right|^{2}}{\|f\|^{2}}(\alpha-(d / n)) \text { so }
$$

$m^{*}[f]$ is a convex combination of the vectors $\alpha-(d / n)$, such that $c_{\alpha} \neq 0$. Thus $m^{*}[f]$ is "in the weight polytope".

(iii) If $f=\Sigma c_{\alpha} m^{\alpha}$ where $c_{\alpha} \neq 0$ implies $c_{\alpha}+\epsilon_{i}-\epsilon_{j}=0$ for $i \neq j$, $1 \leq i, j \leq n$, then $m^{*}[f]$ is diagonal. Here $\epsilon_{i}=(0,0, \ldots, 1$, $0, \ldots, 0)$.

(iv) For $d=n=3, m^{*}\left[\lambda\left(x^{3}+Y^{3}+\mathbf{Z}^{3}\right)+3 \mu X Y Z\right]=0$ for all $\lambda, \mu \in$ C. Thus $\lambda\left(X^{3}+Y^{3}+Z^{3}\right)+3 \mu X Y Z$ is a family of minimal vectors in $\operatorname{Sym}^{3}\left(\mathbf{C}^{3 *}\right)$.

We illustrate the corollary in the case $d=4, n=3$ using variables $X, Y$, and $Z$.

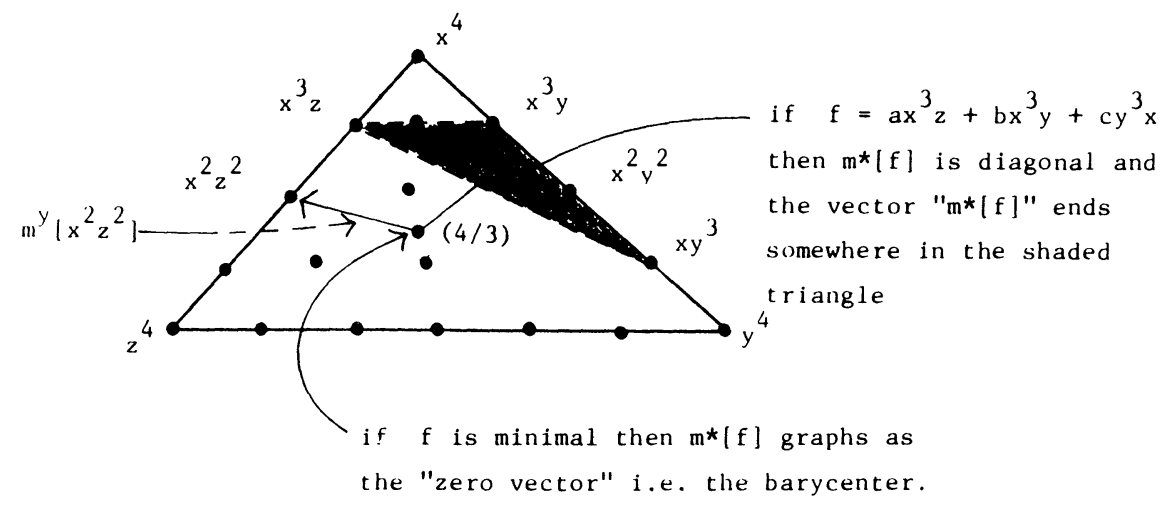

**Parts (i), (ii) and (iii) and their graphic interpretation can be generalized to the moment map arising from an arbitrary representation of a reductive group $G$ on a complex vector space V. 
For arbitrary values of $d$ and $n$, we can find a number of critical points of $\|m\|^{2}: \mathbf{P}(\mathbf{V}) \rightarrow i \mathfrak{k}, \mathbf{V}=\operatorname{Sym}^{d}\left(\mathbf{C}^{n^{*}}\right)$ and determine their index.

LEMMA 10.2 .

(i) Any monomial $m^{\alpha}=x_{n}^{\alpha_{1}} \cdots x^{\alpha_{n}}$ determines a critical point of $\|m\|^{2}: \mathbf{P}(\mathbf{V}) \rightarrow i f$. Furthermore

$$
\begin{aligned}
& \operatorname{index}\left[m^{\alpha}\right]=2 \operatorname{card}\left\{m^{\beta}: \beta \neq \alpha \text { and }\left(\frac{(\alpha-(d / n)}{\|\alpha-(d / n)\|}, \beta-(d / n)\right)\right. \\
&<\|\alpha-(d / n)\|
\end{aligned}
$$

where (,) here denotes the usual inner product on $\mathbf{R}^{n}$

(ii) The critical points with the largest index are the image of a highest weight vector, e.g. $x_{i}^{d}$

$$
\text { index }\left[x_{i}^{d}\right]=2\left(\operatorname{dim}_{\mathbf{C}} \operatorname{Sym}^{d}\left(\mathbf{C}^{n^{*}}\right)-1\right)=2 \operatorname{dim}_{\mathbf{C}} \mathbf{P}(\mathbf{V}) .
$$

Proof. Immediate from Lemma 10.1, and the index formula theorem 6.3

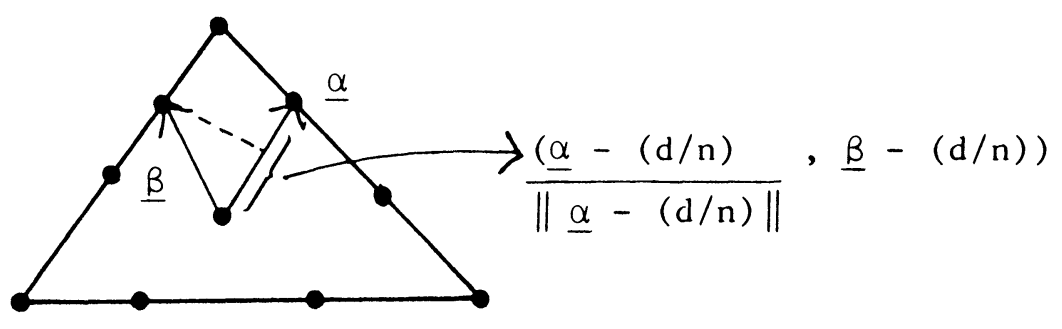

Thus a monomial $m^{b}$ contributes to the index of $m^{\alpha}$ if and only if the projection of $\beta-(d / n)$ in the direction of $\alpha-(d / n)$ is less than $\|\alpha-(d / n)\|$.

Q.E.D

Let $\mathbf{V}_{d, n}=\operatorname{Sym}^{d}\left(\mathbf{C}^{n^{*}}\right)$. There is a natural inclusion $\mathbf{V}_{d, n} \rightarrow \mathbf{V}_{d, n+k}$ for $k \in \mathbf{Z}, k>0$. One may inquire about the image of the critical points and the mininal points. For $\mathbf{V}_{d, n} \rightarrow \mathbf{V}_{d, n+k}$, The old weight polytope, from $\mathbf{V}_{d, n}$, is a face of the new weight polytope (of $\mathbf{V}_{d, n+k}$ ). Denote the respective barycenters by

$$
B_{n}=(\underbrace{d / n, \ldots, d / n}_{n \text {-times }}, \underbrace{0, \ldots, 0}_{k \text { times }})
$$


and $B_{n+K}=(d / n+k, \ldots, d / n+k)$. Then for $f \in \mathbf{V}_{d, n}$, if $m^{*}[f]_{n}$ is diagonal, $m *[f]_{n+k}=\widehat{B_{n+k} B_{n}}+m *[f]_{n}$ (vector sum). Here the subscripts indicate the domain of the dual moment map.

Lemma 10.3.

(i) If $[f] \in \mathbf{P}\left(\mathbf{V}_{d, n}\right)$ is a critical point then $[f] \in \mathbf{P}\left(\mathbf{V}_{d, n+k}\right), k>0$, is a nonminimal critical point. The two indices are related by

$$
\operatorname{index}_{n+k}[f]=\operatorname{index}_{n}[f]+2\left(\operatorname{dim}_{\mathbf{C}}\left(\operatorname{dim}_{\mathbf{C}} \mathbf{V}_{n+k}-\operatorname{dim}_{\mathbf{C}} \mathbf{V}_{n}\right) .\right)
$$

(ii) The gradient flow of $\|m\|_{n+k}^{2}: \mathbf{P}\left(\mathbf{V}_{d, n+k}\right) \rightarrow \mathbf{R}$ on the image $\mathbf{P}\left(\mathbf{V}_{d, n}\right) \rightarrow \mathbf{P}\left(\mathbf{V}_{d, n+k}\right)$ is just the gradient flow of $\|m\|_{n}^{2}:$ $\mathbf{P}\left(\mathbf{V}_{d, n}\right) \rightarrow \mathbf{R}$.

Proof. We may assume $m^{*}[f]_{n}$ is diagonal and $m^{*}[f]_{n}=h-$ $(d / n)$. Then $m^{*}[f]_{n+k}=\left(h_{1}, \ldots, h_{n}, 0, \ldots, 0\right)-(d / n+k)$. But $[f]$ critical for $m^{*}[]_{n}$ implies exp th fixes $[f] \in \mathbf{P}\left(\mathbf{V}_{d, n}\right)$ which implies $\exp t m^{*}[f]_{n+k}$ fixes $[f] \in \mathbf{P}\left(\mathbf{V}_{d, n+k}\right)$, so $[f]$ remains a critical point. The nonminimality of $[f]$ in the bigger space follows from $\|m\|_{n+k}[f]>$ $\left\|\overrightarrow{B_{n+k} \vec{B}_{n}}\right\|>0$ for $k>0$. By the next formula (theorem 6.3)

$$
\operatorname{index}_{n+k}[f]=\operatorname{index}_{n}[f]+\operatorname{card}\left\{m^{\alpha}: \alpha=\alpha_{1}, \ldots, \alpha_{n+k}\right),
$$

some $\alpha_{i} \neq 0$ for $i>n$ so

$$
\operatorname{index}_{n+k}[f]=\operatorname{index}_{n}[f]+2\left(\operatorname{dim}_{\mathbf{C}} \mathbf{V}_{d, n+k}-\operatorname{dim}_{C} \mathbf{V}_{d, n}\right) .
$$

Part (ii) follows from the fact that

$$
\operatorname{grad}\|m\|^{2}(x)=\operatorname{grad} m_{\alpha}(x), \quad \alpha=m^{*}(x) . \quad \text { Q.E.D. }
$$

The lemma implies that one of the projective varieties $C_{M, \alpha / G}$ from the nullcone of $\operatorname{Sym}^{d}\left(\mathbf{C}^{n^{*}}\right)$ will be $\operatorname{Sym}^{d}\left(\mathbf{C}^{n-1 *}\right)_{s s / S L(n-1, \mathbf{C})}$. For the cases of $\operatorname{Sym}^{d}\left(\mathbf{C}^{2 *}\right)$ and $\operatorname{Sym}^{3}\left(\mathbf{C}^{3 *}\right)$ we have computed all of the critical points.

Lemma 10.4. Let $\mathbf{V}=\operatorname{Sym}^{d}\left(\mathbf{C}^{2 *}\right)$, so $\mathbf{P}(\mathbf{V}) \approx \mathbf{P}^{d}$. Then every $K$ coset of nonminimal critical points contain a monomial.

Proof. Suppose $[f]$ is a nonminimal critical point. After replacing $f$ by $f \circ k$, perhaps, we may assume $m *[f]$ is a diagonal matrix. Thus $m *[f]$ is a nonzero multiple of $\alpha=\left[\begin{array}{rr}1 & 0 \\ 0 & -1\end{array}\right]$. Hence $\exp t \alpha$ fixes $[f]$. But $f=$ 
$\sum_{i=0}^{n} c_{i} X^{i} Y^{d i}$ and $\exp t \alpha \cdot X^{i} Y^{d-i}=e^{(2 i-d) t} X^{i} Y^{d-i}$. Thus exp $t \alpha$ acts by a different weight on each monomial so if $[f]$ is to be fixed by $\exp t \alpha,[f]=$ $\left[X^{i} Y^{d-i}\right]$ for some $i$.

Q.E.D.

Thus the projective varieties $C_{M, \alpha / G}$ arising from the stratification of the nullcone for $\mathbf{P}\left(\operatorname{Sym}^{d} \mathbf{C}^{2 *}\right) \approx \mathbf{P}^{d}$ are points. The correspondence is

$x^{i} y^{j}, i<j \leftrightarrow C_{M, \alpha / G} M=\frac{j-i}{2}, \alpha=m^{*}\left[x^{i} y^{j}\right]=2\left[\begin{array}{ll}i-d / 2 & 0 \\ 0 & j-d / 2\end{array}\right]$.

Lemma 10.5. (The critical points of $\|m\|^{2}$ for plane cubic curves). Let $\mathbf{V}=\operatorname{Sym}^{3}\left(\mathbf{C}^{3 *}\right)$. Let $G=\operatorname{SL}(3, \mathbf{C})$. Each $K$-coset of critical points of $\|m\|^{2}: \mathbf{P}(\mathbf{V}) \rightarrow \mathbf{R}$ contains an $[f]$ from the following list. The f's listed have diagonal moment matrices.

\begin{tabular}{|l|c|l|}
\hline \multicolumn{1}{|c|}{$F$} & Index & \multicolumn{1}{|c|}{$m^{*}[f]$ eigenvalues = diagonal entries } \\
\hline$X^{3}$ & 9 & $(3,0,0)-(1,1,1)=(2,-1,-1)$ \\
$X^{2} Y$ & 8 & $(2,1,0)-(1,1,1)=(1,0,-1)$ \\
$X^{2} Z+Y^{2} X$ & 6 & $(3 / 2,1,1 / 2)-(1,1,1)=(1 / 2,0,-1 / 2)$ \\
and $f=\Sigma_{i=0}^{d} c_{i} X^{i} Y^{3-i}$ & 6 & $(3 / 2,3 / 2,0)-(1,1,1)=(1 / 2,1 / 2,-1)$ \\
$\quad$ which is minimal & & \\
in Sym ${ }^{3}\left(\mathbf{C}^{2 *}\right)^{* *}$ & & \\
$3 X^{2} Z+2 Y^{3}$ & 5 & $1 / 13(5,-1,-4)$ \\
$\lambda\left(X^{3}+Y^{3}+Z^{3}\right)+3 \mu X Y Z$ & 0 & $(0,0,0)$ \\
\hline
\end{tabular}

Proof. One can check that the $f$ 's listed are critical points by noting that $\exp t m^{*}[f]$ fixes $[f]$. One can also check their index from the index formula. To see that these are all of the critical points, one can simply write down all of the cubics $g$ that are fixed by a diagonal one parameter subgroup exp $\alpha t$. Then one calculates $m^{*}[g]$. If $m^{*}[g]$ is a multiple of $\alpha, g$ is a critical point. To do these calculations, it is very helpful to use the weight diagram as follows

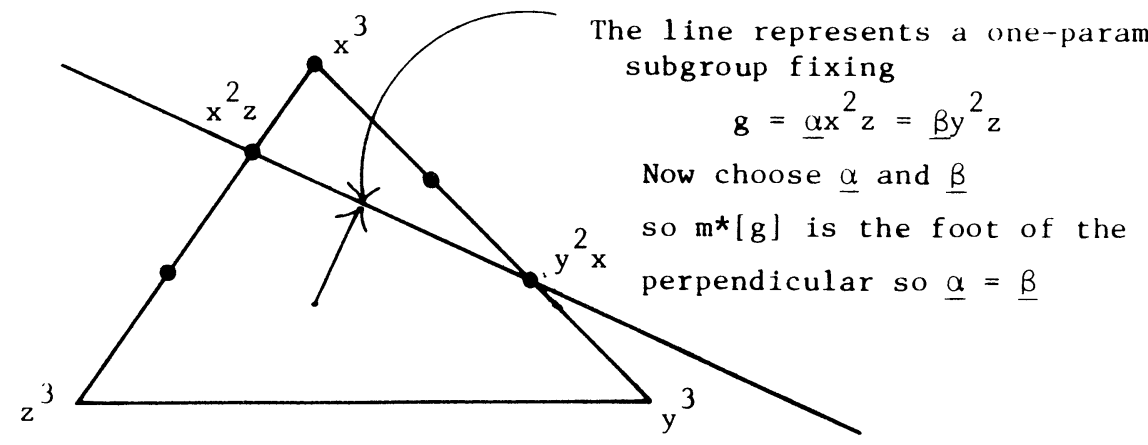


Now one can check ** and find that up to $K$-equivalence, $x^{3}+y^{3}$ is only one minimal cubic $\Sigma_{i=0}^{d} c_{i} x^{i} y^{3-i}$. Hence the projective varieties $C_{M, \alpha / G}$ arising from the stratification of the null cone for $\mathbf{P}\left(\mathrm{Sym}^{3} \mathbf{C}^{3^{*}}\right)$ are again points and the chart shows that there are 5 points, $C_{M, \alpha / G}$.

For $\mathbf{P}\left(\mathrm{Sym}^{3} \mathbf{C}^{4 *}\right)$, "quartics in 3 variables", though, not all of the varieties $C_{M, \alpha / G}$ will be points. For there will be one strata with $C_{M, \alpha}$ equal to the orbits of $\lambda\left(x^{3}+y^{3}+2^{3}\right)+3 \mu x y z, \lambda, \mu \in \mathbf{C}$, since every minimal cubic in 3 variables is $K$-equivalent to one of these. Thus this variety $C_{M, \alpha / G}$ will have dimension 1 and will be singular at "the two cubics" with complex multiplication and "at $x y z$ ".

\section{REFERENCES}

[A] Arnol'd, V., Mathematical Methods of Classical Mechanics, Springer-Verlag, 1978.

[A-B] Atiyah, M., and Bott, R., The Yang-Mills equations over Riemann surfaces, to appear.

[At] Atiyah, M., Convexity and commuting Hamiltonians, Bull. London Math. Soc., 14 (1982), 1-15.

[G-S] Guillemin, Y. and Sternberg, S., Geometric Quantizations and multiplicities of group representations, to appear.

,Guillemin, V. and Sternberg, S., Convexity properties of the moment mapping, to appear.

[He] Heckman, G. J., Projections of Orbits and Asymptotic Behaviour of Multiplicities for Compact Groups, thesis, Leiden, 1980.

[H] Hesselink, W., Desingularizations of varieties of null forms, Inventiones Math., 55 (1979), 141-163.

[M] Mumford, D., Geometric Invariant theory, Springer-Verlag, New York, 1965.

[K] Kempf, Instability in invariant theory, Annals of Math., 108 (1978), 299-317.

[K-N] Kempf, G. and Ness, L., The length of vectors in representation spaces, Springer Lecture Notes \#732, Algebraic Geometry, Proceedings, Copenhagen, 1978, 233-244.

[N] Ness, L., Mumford's numerical function and stable projective hypersurfaces, Springer Lecture Notes \#732 Algebraic Geometry, Proceedings, Copenhagen, $1978,417-454$. 


\title{
APPENDIX: PROOF OF THE CONVEXITY THEOREM
}

\author{
By David Mumford
}

In this Appendix, we prove the convexity theorem of Section 5 by using the techniques of geometric invariant theory. Half of the proof in fact can be viewed as giving a purely algebraic definition of the convex set Image $(\check{m})$. First fix the following notation:

$G$ a reductive algebraic group, Lie algebra $g$

$K$ a maximal compact subgroup, Lie algebra $¥$

$T$ a maximal torus such that $T=$ complexification of $K \cap T$

$B \quad$ a Borel subgroup containing $T$

$t \quad$ the Lie algebra of $T$

$\mathrm{t}^{+}$the positive Weyl chamber determined by $B$

V a representation space for $G$

$\langle$,$\rangle an inner product on \mathrm{g}$ satisfying (12).

As in Section 5, we get moment maps

$$
\begin{aligned}
& m^{*}: \mathbf{P}(V) \rightarrow i \mathfrak{f} \\
& \check{m}: \mathbf{P}(V) \rightarrow i \mathrm{t}^{+} \text {. }
\end{aligned}
$$

Let $X \subset \mathbf{P}(V)$ be a $G$-invariant subvariety. We wish to prove that $\check{m}(X)$ is a convex rational polytope in $i t^{+}$. The proof consists in 2 steps.

Step I: Let $w \in$ Weyl group carry $\mathrm{t}^{+}$to $-\mathrm{t}^{+}$and let an integral point (in the "weight" lattice) $\alpha \in \mathrm{t}$ (define a character of $T$, hence a line bundle $L^{\alpha}$ on $G / B$. Then I claim:

$\forall \alpha \in i \mathrm{t}^{+}, \alpha=\beta / n, \beta$ integral

$(-w) \alpha \in \check{m}(x) \Leftrightarrow$ the generic point of $X \times G / B$ is $\mathcal{O}_{x}(n) \otimes$ $L^{\beta}$-semi-stable.

Step II: Let $G$ act on $\mathbf{V}^{(1)}, \ldots, \mathbf{V}^{(m)}$ and let $X \subset \mathbf{P}\left(\mathbf{V}^{(1)}\right) \times \cdots \times$ $\mathbf{P}\left(\mathbf{V}^{(m)}\right)$ be a $G$-invariant subvariety. Then there is a rational convex polyhedral cone $C \subset \mathbf{R}_{+}^{m}$ such that for all $k_{i} \in \mathbf{Z}, k_{i} \geq 0$, 
(A2) $\quad\left(k_{1}, \ldots, k_{m}\right) \in C \Leftrightarrow$ the generic point of $x$ is $\mathcal{O}_{\mathbf{p}}\left(k_{1}\right) \otimes \cdots \otimes$ $\mathcal{\Theta}_{\mathbf{P}}\left(k_{m}\right)$-semi-stable.

(A1) and (A2) together prove that the rational points in $\check{m}(X)$ are the rational points in a rational convex polytope. In view of lemma $2.1, m$ and hence $\check{m}$ is generically regular and hence rational points will be dense in $\check{m}(X)$, unless the intersection of $K$ and the generic stabilizer $G_{x}$ is positive dimensional. In the rather special case that $\operatorname{dim} K \cap G_{x}>0$, all $x$, we can still argue that rational points are dense in $\check{m}(x)$ as follows: Replacing $x$ by $k x$, some $k \in x$, we may assume $m(x) \in i$ t* and apply lemmas 1.2 and 2.1 for the moment map just for the torus $T$. By lemma 1.2, the components of $m$ in directions in $T \cap G_{x}$ are rational and constant on $T \cdot x$; by lemma 2., the other components vary independently hence can be made rational by replacing $x$ by $t \cdot x, t \in T$ arbitrarily close to $e$.

Proof of Step I. This follows from 2 easy lemmas:

Lemma A3. Embed $\mathbf{P}\left(\mathbf{V}_{1}\right) \times \mathbf{P}\left(\mathbf{V}_{2}\right)$ in $\mathbf{P}\left(\mathbf{V}_{1} \otimes \mathbf{V}_{2}\right)$ as usual. Then the moment map for $\mathbf{P}\left(\mathbf{V}_{1}\right) \times \mathbf{P}\left(\mathbf{V}_{2}\right)$ is the sum of the moment maps for $\mathbf{P}\left(\mathbf{V}_{1}\right), \mathbf{P}\left(\mathbf{V}_{2}\right)$ :

$$
m_{\mathbf{P}\left(\mathbf{v}_{1} \otimes \mathbf{v}_{2}\right)}(x \otimes y)=m_{\mathbf{P}\left(\mathbf{v}_{1}\right)}(x)+m_{\mathbf{P}\left(\mathbf{v}_{2}\right)}(y)
$$

This follows from the formula of Section 1.

Lemma A4. Let $\alpha \in \mathrm{t}^{+}$be integral and let it define $L^{\alpha}$ on $G / B$. Map $G / B$ to $\mathbf{P}(\mathbf{V}), \mathbf{V}=\Gamma\left(G / B, L^{\alpha}\right)$ : call this $\phi: G / B \rightarrow \mathbf{P}(\mathbf{V})$. Then

$$
m_{\mathbf{P}(\mathbf{V})(\phi(G / B))}^{*}=K \text {-orbit of } i \alpha \text { in } i \cdot \mathfrak{f}
$$

This follows from the calculations of Section 8. Putting these together, we see that

$$
\begin{aligned}
\begin{array}{r}
\text { gen. pt. of } X \times G / B \text { is } \\
\mathcal{O}_{x}(n) \otimes L^{\beta} \text {-semi-stable } \begin{array}{r}
\text { Image }\left(m_{X \times G / B}\right) \text { where } x \times G / B \text { is } \\
\text { mapped to } \mathbf{P}(\mathbf{V}) ; \mathbf{V}=\Gamma\left(\mathcal{O}_{x}(n)\right) \otimes \\
\Gamma\left(L^{\beta}\right)
\end{array} \\
\Leftrightarrow \exists x \in X, y \in G / B \text { such that } n \cdot m_{x}(x)+ \\
m_{G / B}(y)=0
\end{array} \\
\Leftrightarrow \exists x \in X \text { such that } n \cdot m_{x}(x)+\beta=0 \\
\Leftrightarrow \exists x \in X \text { such that } n \cdot m_{x}(x)+\omega(\beta)=0 \\
\quad \text { or } m_{x}(x)=(-\omega)(\alpha) . \quad \text { Q.E.D. }
\end{aligned}
$$


Proof of Step II. Decompose $\mathbf{V}^{(\alpha)}$, into weight spaces with respect to $T:$

$$
\mathbf{V}^{(\alpha)}=\underset{i \in W_{\alpha}}{\oplus} \mathbf{V}^{(\alpha)}, \quad W_{\alpha} \subset \mathfrak{t}^{*}
$$

For each $x \in X$, write the coordinates of $x$ in the Segre embedding as

$$
\left(\sum_{i \in W_{1}} v_{i}^{(1)}\right) \otimes \cdots \otimes\left(\sum_{i \in W_{m}} v_{i}^{(m)}\right), \quad v_{i}^{(\alpha)} \in \mathbf{V}_{i}^{(\alpha)}
$$

Define

$$
S_{\alpha}(x)=\left\{i \in W_{\alpha} \mid v_{i}^{(\alpha)} \neq 0\right\}
$$

and call the $m$-tuples of subsets $\left(S_{1}(x), \ldots, S_{n}(x)\right)$ the " $T$-state" of the point $x$. As there are only finitely many possible $T$-states, $X$ decomposes into locally closed pieces

$$
X=\Pi X_{\ell}
$$

where all points on $X_{\ell}$ have the same $T$-state. For each $\ell$, either $G \cdot X_{\ell}$ is dense in $X$ or is part of a finite set of proper subvarieties. Call the $T$-states of the $X_{\ell}$ such that $G \cdot X_{\ell}$ is dense the generic $T$-states, and write then $\left.(S)_{1 \beta}, \ldots, S_{n \beta}\right), \beta \in I$. We now apply the numerical criterion of semistability of Geometric Invariant Theory:

$x \in X$ semi-stable $\Leftrightarrow \forall g \in G, \forall$ one-parameter subgroups $\lambda \in \Gamma(T)$,

$$
\mu(g \cdot x, \lambda) \geq 0
$$

If semi-stability is calculated by the embedding $\mathcal{O}_{\mathbf{P}}\left(k_{1}\right) \otimes \cdots \otimes \vartheta_{\mathbf{P}}\left(k_{m}\right)$, then

$$
\mu(g \cdot x, \lambda)=\max _{\substack{s_{1} \in S_{1}(g x) \\ ﹎{m} \\ s_{m} \in S_{m}(g x)}} \ell\left(\sum_{\alpha=1}^{m} k_{\alpha} s_{\alpha}\right)
$$

where $\lambda$ corresponds to $\ell \in \mathrm{t}$. Therefore: 
the gen. pt. $x \in X$ is $\vartheta_{\mathbf{P}}\left(k_{1}\right) \otimes \cdots \otimes \mathcal{\Theta}_{\mathbf{P}}\left(k_{m}\right)-$ semi-stable
$\Leftrightarrow \forall$ generic $T$-states $\beta, \forall \ell \in \mathrm{t}$

To analyze the right hand side, define

$$
\mathcal{S}_{\beta}^{*} \subset \mathbf{R}^{m} \times \mathrm{t}^{*}
$$

by

$$
\mathcal{S}_{\beta}^{*}=\left\{\left(k_{1}, \ldots, k_{m}, \Sigma k_{\alpha} s_{\alpha}\right) \mid k_{i} \in \mathbf{R}, k_{i} \geq 0, s_{\alpha} \in \text { convex hull }\left(\mathcal{S}_{\alpha \beta}\right)\right.
$$

Then $S_{\beta}^{*}$ is rational convex polyhedral cone such that

$$
S_{\beta}^{*} \cap\left[\left(k_{1}, \ldots, k_{m}\right) \times \mathrm{t}^{*}\right]=\sum_{\alpha} k_{\alpha}\left(\text { convexhull of } \delta_{\alpha \beta}\right) .
$$

The condition on the right hand side is just that for all $\beta$,

$$
0 \in \sum_{\alpha} k_{\alpha}\left(\text { convexhull of } S_{\alpha \beta}\right)
$$

But $S_{\beta}^{*}$ may be defined by a finite set of inequalities

$$
\left(k_{1}, \ldots, k_{m}, x\right) \in \mathcal{S}_{\beta}^{*} \Leftrightarrow \sum_{\alpha=1} e_{\alpha \beta \gamma} k_{\alpha}+\ell_{\beta \gamma}(x) \geq 0,
$$

hence it follows that the right hand side is equivalent to $\Sigma_{\alpha} e_{\alpha \beta \gamma} k_{\alpha} \geq 0$, all $\beta, \gamma$ which defines a rational convex polyhedral cone.

Q.E.D. 